\title{
Mixed quantum-classical dynamics on the exact time-dependent potential energy surface: A fresh look at non-adiabatic processes
}

\author{
Federica Agostini, ${ }^{1}$ Ali Abedi, ${ }^{1}$ Yasumitsu Suzuki, ${ }^{1}$ and E. K. U. Gross ${ }^{1}$ \\ ${ }^{1}$ Max-Planck Institut für Mikrostrukturphysik, Weinberg 2, D-06120 Halle, Germany
}

\begin{abstract}
The exact nuclear time-dependent potential energy surface arises from the exact decomposition of electronic and nuclear motion, recently presented in [A. Abedi, N. T. Maitra, and E. K. U. Gross, Phys. Rev. Lett. 105, 123002 (2010)]. Such time-dependent potential drives nuclear motion and fully accounts for the coupling to the electronic subsystem. We investigate the features of the potential in the context of electronic non-adiabatic processes and employ it to study the performance of the classical approximation on nuclear dynamics. We observe that the potential, after the nuclear wave-packet splits at an avoided crossing, develops dynamical steps connecting different regions, along the nuclear coordinate, in which it has the same slope as one or the other adiabatic surface. A detailed analysis of these steps is presented for systems with different non-adiabatic coupling strength. The exact factorization of the electron-nuclear wave-function is at the basis of the decomposition. In particular, the nuclear part is the true nuclear wave-function, solution of a time-dependent Schroedinger euqation and leading to the exact many-body density and current density. As a consequence, the Ehrenfest theorem can be extended to the nuclear subsystem and Hamiltonian, as discussed here with an analytical derivation and numerical results.
\end{abstract}

\section{INTRODUCTION}

The Born-Oppenheimer (BO) [1], or adiabatic, treatment of the coupled motion of electrons and nuclei is among the most fundamental approximations in modern condensed-matter theory and forms the basis of our understanding of dynamical processes in molecules and solids. It offers a practical way to visualize a molecule or solid as a set of nuclei moving on a single potential energy surface (PES) generated by the electrons in a given eigenstate. However, it is based on the assumption that the electrons adjust instantaneously to adiabatic changes of the nuclear positions, and a variety of interesting phenomena in physics, chemistry and biology take place in the regime where this approximation breaks down. Prominent examples are the process of vision [2--4], photo-synthesis [5, 6], photo-voltaic processes [7--9], proton-transfer/hydrogen storage [10-13] as well as phonon-induced superconductivity.

Non-adiabatic molecular processes are usually explained in terms of BOPESs and transitions between the $\mathrm{BO}$ electronic states. In this context, the solution of the time-dependent Schrödinger 
equation (TDSE) is expanded in the complete system of BO electronic states, leading to a nuclear wave-packet with contributions on several BOPESs that undergo transitions in the regions of strong non-adiabatic coupling. This approach provides a formally exact description of the complete system if all the electronic states are taken into account. However, practical applications are limited to a small number of degrees of freedom. For large systems, the only feasible way of dealing with non-adiabatic processes is the introduction of classical or semi-classical approximations for the nuclear motion, coupled, non-adiabatically, to the (quantum mechanical) electrons. Although widely investigated [14-17], the nature of the force driving the classical nuclei in this mixed quantum-classical treatment has not yet been fully identified.

Recently [18], this problem has been addressed from a novel perspective by referring to the exact representation of the full molecular wave-function [19, 20] as a single product of a purely nuclear wave-function and an electronic factor that parametrically depends on the nuclear coordinates. In this framework, a TDSE for the nuclear wave-function is derived, where a timedependent potential energy surface (TDPES) and a time-dependent vector potential arise as exact concepts and provide the driving force for the nuclear evolution.

The present paper discusses situations where the vector potential can be set to zero by an appropriate choice of gauge, thus leaving the TDPES as the only potential responsible for the nuclear dynamics. In this case, the force on the nuclei, in a classical sense, can be obtained as the gradient of the TDPES. But, is this the true classical force on the nuclei? We will try to address this issue by employing the exact TDPES, that is known for the simple system studied here, for the propagation of classical trajectories in order to (i) examine the quality of the classical approximation for the nuclear motion and (ii) get insight into the properties of approximated classical forces for an eventual mixed quantum-classical treatment of non-adiabatic processes. Moreover, we will discuss the connections [18] between such novel approach, based on a single TDPES, and the well-established description in terms of several static (coupled) BOPESs.

The paper is organized as follows. In Section II the exact factorization of the time-dependent electron-nuclear wave-function is presented and the equations that govern the evolution of the electronic and nuclear subsystems are discussed. The TDPES is investigated and analyzed in detail in Section III for systems showing different degree of non-adiabaticity. Section IV]presents some results obtained by performing classical dynamics on the exact surface and in Section $V$ we discuss the Ehrenfest theorem in the exact factorization representation of the full wave-function. In Section $\mathrm{VI}$ some concluding words are given. 


\section{EXACT DECOMPOSITION OF THE ELECTRONIC AND NUCLEAR MOTION}

In the absence of a time-dependent external field, a system of interacting electrons and nuclei is described, non-relativistically, by the Hamiltonian

$$
\hat{H}=\hat{T}_{n}+\hat{H}_{B O},
$$

where $\hat{T}_{n}$ is the nuclear kinetic energy operator and

$$
\hat{H}_{B O}(\underline{\underline{\mathbf{r}}}, \underline{\underline{\mathbf{R}}})=\hat{T}_{e}(\underline{\underline{\mathbf{r}}})+\hat{W}_{e e}(\underline{\underline{\mathbf{r}}})+\hat{V}_{e n}(\underline{\underline{\mathbf{r}}}, \underline{\underline{\mathbf{R}}})+\hat{W}_{n n}(\underline{\underline{\mathbf{R}}}),
$$

is the standard BO electronic Hamiltonian. The symbols $\underline{\underline{\mathbf{r}}}$ and $\underline{\underline{\mathbf{R}}}$ are used to collectively indicate the coordinates of $N_{e}$ electrons and $N_{n}$ nuclei, respectively. It has been proved in [19, 20] that the full time-dependent electron-nuclear wave function, $\Psi(\underline{\underline{\mathbf{r}}}, \underline{\underline{\mathbf{R}}}, t)$, that is the solution of the TDSE,

$$
\hat{H} \Psi(\underline{\underline{\mathbf{r}}}, \underline{\underline{\mathbf{R}}}, t)=i \hbar \partial_{t} \Psi(\underline{\underline{\mathbf{r}}}, \underline{\underline{\mathbf{R}}}, t),
$$

can be exactly factorized to the correlated product,

$$
\Psi(\underline{\underline{\mathbf{r}}}, \underline{\underline{\mathbf{R}}}, t)=\chi(\underline{\underline{\mathbf{R}}}, t) \Phi_{\underline{\underline{\mathbf{R}}}}(\underline{\underline{\mathbf{r}}}, t),
$$

of the nuclear wave-function, $\chi(\underline{\underline{\mathbf{R}}}, t)$, and the electronic wave-function, $\Phi_{\underline{\underline{\mathbf{R}}}}(\underline{\underline{\mathbf{r}}}, t)$, that parametrically depends on the nuclear configuration and satisfies the partial normalization condition $(\mathrm{PNC})$,

$$
\int d \underline{\underline{\mathbf{r}}}\left|\Phi_{\underline{\mathbf{R}}}(\underline{\underline{\mathbf{r}}}, t)\right|^{2}=1, \quad \forall \underline{\underline{\mathbf{R}}}, t .
$$

The PNC is an essential element of this representation. Without imposing the PNC, the full wavefunction can be factorized in many different (unphysical) ways. It is the PNC that makes the factorization (4) unique up to within a $(\underline{\underline{\mathbf{R}}}, t)$-dependent gauge transformation,

$$
\begin{aligned}
& \chi(\underline{\underline{\mathbf{R}}}, t) \rightarrow \tilde{\chi}(\underline{\underline{\mathbf{R}}}, t)=e^{-\frac{i}{\hbar} \theta(\underline{\underline{\mathbf{R}}}, t)} \chi(\underline{\underline{\mathbf{R}}}, t) \\
& \Phi_{\underline{\underline{\mathbf{R}}}}(\underline{\underline{\mathbf{r}}}, t) \rightarrow \tilde{\Phi}_{\underline{\underline{\mathbf{R}}}}(\underline{\underline{\mathbf{r}}}, t)=e^{\frac{i}{\hbar} \theta(\underline{\underline{\mathbf{R}}}, t)} \Phi_{\underline{\underline{\mathbf{R}}}}(\underline{\underline{\mathbf{r}}}, t) .
\end{aligned}
$$

Another important implication of imposing the PNC is that the diagonal of the $N$-body nuclear density matrix of the complete system is equal to $|\chi(\underline{\underline{\mathbf{R}}}, t)|^{2}$.

The stationary variations [21] of the quantum mechanical action[26] w.r.t. $\Phi_{\underline{\underline{\mathbf{R}}}}(\underline{\underline{\mathbf{r}}}, t)$ and $\chi(\underline{\underline{\mathbf{R}}}, t)$ lead to the derivation of the equations of motion

$$
\begin{aligned}
& \left(\hat{H}_{B O}(\underline{\underline{\mathbf{r}}}, \underline{\underline{\mathbf{R}}})+\hat{U}_{e n}^{\text {coup }}\left[\Phi_{\underline{\mathbf{R}}}, \chi\right]-\epsilon(\underline{\underline{\mathbf{R}}}, t)\right) \Phi_{\underline{\underline{\mathbf{R}}}}(\underline{\underline{\mathbf{r}}}, t)=i \hbar \partial_{t} \Phi_{\underline{\underline{\mathbf{R}}}}(\underline{\underline{\mathbf{r}}}, t) \\
& \left(\sum_{\nu=1}^{N_{n}} \frac{\left[-i \hbar \nabla_{\nu}+\mathbf{A}_{\nu}(\underline{\underline{\mathbf{R}}}, t)\right]^{2}}{2 M_{\nu}}+\epsilon(\underline{\underline{\mathbf{R}}}, t)\right) \chi(\underline{\underline{\mathbf{R}}}, t)=i \hbar \partial_{t} \chi(\underline{\underline{\mathbf{R}}}, t) .
\end{aligned}
$$


Here, $\epsilon(\underline{\underline{\mathbf{R}}}, t)$ is the TDPES, defined as

$$
\epsilon(\underline{\underline{\mathbf{R}}}, t)=\left\langle\Phi_{\underline{\underline{\mathbf{R}}}}(t)\left|\hat{H}_{B O}+\hat{U}_{\text {en }}^{\text {coup }}-i \hbar \partial_{t}\right| \Phi_{\underline{\underline{\mathbf{R}}}}(t)\right\rangle_{\underline{\underline{\mathbf{r}}}},
$$

$\hat{U}_{\text {en }}^{\text {coup }}\left[\Phi_{\underline{\underline{\mathbf{R}}}}, \chi\right]$ is what we name "electron-nuclear coupling operator", defined as

$$
\begin{aligned}
\hat{U}_{\text {en }}^{\text {coup }}\left[\Phi_{\underline{\underline{\mathbf{R}}}}, \chi\right]= & \sum_{\nu=1}^{N_{n}} \frac{1}{M_{\nu}}\left[\frac{\left[-i \hbar \nabla_{\nu}-\mathbf{A}_{\nu}(\underline{\underline{\mathbf{R}}}, t)\right]^{2}}{2}\right. \\
& \left.+\left(\frac{-i \hbar \nabla_{\nu} \chi}{\chi}+\mathbf{A}_{\nu}(\underline{\underline{\mathbf{R}}}, t)\right)\left(-i \hbar \nabla_{\nu}-\mathbf{A}_{\nu}(\underline{\underline{\mathbf{R}}}, t)\right)\right],
\end{aligned}
$$

and $\mathbf{A}_{\nu}(\underline{\underline{\mathbf{R}}}, t)$ is the time-dependent vector potential potential,

$$
\mathbf{A}_{\nu}(\underline{\underline{\mathbf{R}}}, t)=\left\langle\Phi_{\underline{\underline{\mathbf{R}}}}(t) \mid-i \hbar \nabla_{\nu} \Phi_{\underline{\underline{\mathbf{R}}}}(t)\right\rangle_{\underline{\underline{\mathbf{r}}}} .
$$

The symbol $\langle\cdot\rangle_{\underline{\underline{r}}}$ indicates an integration over electronic coordinates only.

In Eqs. (7) and (8), $\hat{U}_{e n}^{\text {coup }}\left[\Phi_{\underline{\mathbf{R}}}, \chi\right], \epsilon(\underline{\underline{\mathbf{R}}}, t)$ and $\mathbf{A}_{\nu}(\underline{\underline{\mathbf{R}}}, t)$ mediate the coupling between the electronic and nuclear motions in a formally exact way. The electron-nuclear coupling operator, $\hat{U}_{\text {en }}^{\text {coup }}\left[\Phi_{\underline{\underline{\mathbf{R}}}}, \chi\right]$, in the electronic equation $(7)$, depends on the nuclear wave-function and the first and second derivatives of the electronic wave-function with respect to the nuclear coordinates. This operator includes the coupling to the nuclear subsystem beyond the parametric dependence in the BO Hamiltonian $\hat{H}_{B O}(\underline{\underline{\mathbf{r}}}, \underline{\underline{\mathbf{R}}})$. The nuclear equation $(8)$, on the other hand, has a particularly appealing form of a Schrödinger equation that contains a time-dependent vector potential (11) and a time-dependent scalar potential (9) that uniquely [27] govern the nuclear dynamics and yield the nuclear wave-function. $\chi(\underline{\underline{\mathbf{R}}}, t)$ is interpreted as the nuclear wave-function since it leads to an $N$-body nuclear density, $\Gamma(\underline{\underline{\mathbf{R}}}, t)=|\chi(\underline{\underline{\mathbf{R}}}, t)|^{2}$, and an $N$-body current density, $\mathbf{J}_{\nu}(\underline{\underline{\mathbf{R}}}, t)=\operatorname{Im}\left(\chi^{*} \nabla_{\nu} \chi\right)+\Gamma(\underline{\underline{\mathbf{R}}}, t) \mathbf{A}_{\nu}$, which reproduce the true nuclear $N$-body density and current density obtained from the full wave-function $\Psi(\underline{\underline{\mathbf{r}}}, \underline{\underline{\mathbf{R}}}, t)$ [20]. The uniqueness of $\epsilon(\underline{\underline{\mathbf{R}}}, t)$ and $\mathbf{A}_{\nu}(\underline{\underline{\mathbf{R}}}, t)$ can be straightforwardly proved by following the steps of the current-density version [22] of the Runge-Gross theorem [23]. The scalar potential and the vector potential transform as

$$
\begin{aligned}
\tilde{\epsilon}(\underline{\underline{\mathbf{R}}}, t) & =\epsilon(\underline{\underline{\mathbf{R}}}, t)+\partial_{t} \theta(\underline{\underline{\mathbf{R}}}, t) \\
\tilde{\mathbf{A}}_{\nu}(\underline{\underline{\mathbf{R}}}, t) & =\mathbf{A}_{\nu}(\underline{\underline{\mathbf{R}}}, t)+\nabla_{\nu} \theta(\underline{\underline{\mathbf{R}}}, t)
\end{aligned}
$$

under the gauge transformation (6). 


\section{TIME-DEPENDENT POTENTIAL ENERGY SURFACE}

In this work, we present a detailed study of the TDPES for strongly coupled electronic and nuclear motions. In order to obtain the TDPES, the full electron-nuclear wave-function has to be calculated. Therefore, we need to choose a system that is simple enough to allow for a numerically exact treatment and that nevertheless exhibits characteristic features associated with non-adiabatic dynamics. Here, we employ the model of Shin and Metiu [24], consisting of three ions and a single electron, as depicted in Fig. 1. Two ions are fixed at a distance of $L=19.0 a_{0}$, the

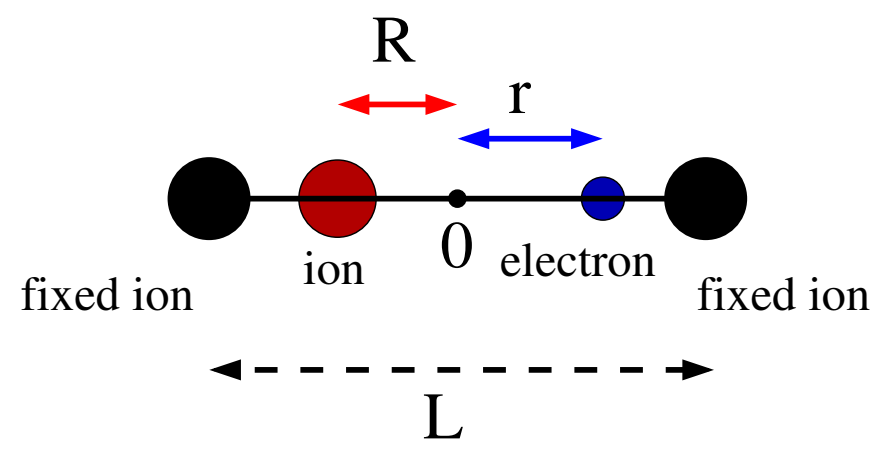

FIG. 1: Schematic representation of the model system described by the Hamiltonian (14). $R$ and $r$ indicate the coordinates of the moving ion and electron, respectively, in one dimension. $L$ is the distance between the fixed ions.

third ion and the electron are free to move in one dimension along the line joining the two fixed ions. The Hamiltonian of this system reads

$$
\begin{aligned}
\hat{H}(r, R)= & -\frac{1}{2} \frac{\partial^{2}}{\partial r^{2}}-\frac{1}{2 M} \frac{\partial^{2}}{\partial R^{2}}+\frac{1}{\left|\frac{L}{2}-R\right|}+\frac{1}{\left|\frac{L}{2}+R\right|}-\frac{\operatorname{erf}\left(\frac{|R-r|}{R_{f}}\right)}{|R-r|} \\
& -\frac{\operatorname{erf}\left(\frac{\left|r-\frac{L}{2}\right|}{R_{r}}\right)}{\left|r-\frac{L}{2}\right|}-\frac{\operatorname{erf}\left(\frac{\left|r+\frac{L}{2}\right|}{R_{l}}\right)}{\left|r+\frac{L}{2}\right|} .
\end{aligned}
$$

Here, the symbols $\underline{\underline{\mathbf{r}}}$ and $\underline{\underline{\mathbf{R}}}$ are replaced by $r$ and $R$, the coordinates of the electron and the movable ion measured from the center of the two fixed ions and $M=1836$ is the mass of the movable ion. The parameters $R_{f}, R_{l}$ and $R_{r}$ specify the interactions between the charged particles and can be tuned to have different couplings between the electronic and nuclear motions.

To obtain the TDPES, we first solve the TDSE (3) for the complete system and obtain the full wave-function, $\Psi(r, R, t)$. This is done by the numerical integration of the TDSE using SPOtechnique [25], with the time-steps of $2.4 \times 10^{-3} \mathrm{fs}$ (or 0.1 a.u.). The nuclear density is calculated, 
at each time, as the marginal probability of the configuration $\underline{\underline{\mathbf{R}}}$ [28] from the full wave-function

$$
|\chi(\underline{\underline{\mathbf{R}}}, t)|^{2}=\int d \underline{\underline{\mathbf{r}}}|\Psi(\underline{\underline{\mathbf{r}}}, \underline{\underline{\mathbf{R}}}, t)|^{2} .
$$

The phase $S(\underline{\underline{\mathbf{R}}}, t)$ of $\chi(\underline{\underline{\mathbf{R}}}, t)$ is determined by the choice of the gauge. We use the exact equality

$$
\mathbf{A}_{\nu}(\underline{\underline{\mathbf{R}}}, t)=|\chi(\underline{\underline{\mathbf{R}}}, t)|^{-2} \operatorname{Im} \int d \underline{\underline{\mathbf{r}}} \Psi^{*}(\underline{\underline{\mathbf{r}}}, \underline{\underline{\mathbf{R}}}, t) \nabla_{\nu} \Psi(\underline{\underline{\mathbf{r}}}, \underline{\underline{\mathbf{R}}}, t)-\nabla_{\nu} S(\underline{\underline{\mathbf{R}}}, t)
$$

which follows immediately from the definition (11) of the vector potential by inserting the factorization (4). The gauge is chosen by setting the vector potential to zero $A(R, t) \equiv 0$ in Eq. (16), which is possible in our specific example because we are dealing with a one-dimensional system. Obviously, the choice of the gauge does not affect any physical observable. $S(R, t)$ is thus determined from the expression

$$
S(R, t)=\int^{R} d R^{\prime}\left|\chi\left(R^{\prime}, t\right)\right|^{-2} \operatorname{Im} \int d r \Psi^{*}\left(r, R^{\prime}, t\right) \nabla_{R^{\prime}} \Psi\left(r, R^{\prime}, t\right) .
$$

From the calculated exact nuclear wave-function $\chi(\underline{\underline{\mathbf{R}}}, t)=e^{-\frac{i}{\hbar} S(\underline{\underline{\mathbf{R}}}, t)}|\chi(\underline{\underline{\mathbf{R}}}, t)|$, we obtain the TDPES $\epsilon(\underline{\underline{\mathbf{R}}}, t)$ from Eq. (9) by explicitly calculating the electronic wave-function $\Phi_{\underline{\underline{\mathbf{R}}}}(\underline{\underline{\mathbf{r}}}, t)=$ $\Psi(\underline{\underline{\mathbf{r}}}, \underline{\underline{\mathbf{R}}}, t) / \chi(\underline{\underline{\mathbf{R}}}, t)$. Alternatively, we may invert the nuclear equation (8). In the gauge we have implemented to perform the calculations, the TDPES alone determines the time evolution of $\chi(\underline{\underline{\mathbf{R}}}, t)$. In order to investigate the TDPES in detail, we study its gauge-invariant (GI) and gaugedependent (GD) constituents separately (it can be easily proven that $\epsilon_{G I}(\underline{\underline{\mathbf{R}}}, t)=\epsilon_{G I}(\underline{\underline{\mathbf{R}}}, t)$ and $\tilde{\epsilon}_{G D}(\underline{\underline{\mathbf{R}}}, t)=\epsilon_{G D}(\underline{\underline{\mathbf{R}}}, t)+\partial_{t} \theta(\underline{\underline{\mathbf{R}}}, t)$ under the transformations in Eqs. (6) $)$,

$$
\epsilon(\underline{\underline{\mathbf{R}}}, t)=\epsilon_{G I}(\underline{\underline{\mathbf{R}}}, t)+\epsilon_{G D}(\underline{\underline{\mathbf{R}}}, t),
$$

where

$$
\epsilon_{G I}(\underline{\underline{\mathbf{R}}}, t)=\left\langle\Phi_{\underline{\underline{\mathbf{R}}}}(t)\left|\hat{H}_{B O}\right| \Phi_{\underline{\underline{\mathbf{R}}}}(t)\right\rangle_{\underline{\underline{\mathbf{r}}}}+\sum_{\nu=1}^{N_{n}}\left(\frac{\hbar^{2}}{2 M_{\nu}}\left\langle\nabla_{\nu} \Phi_{\underline{\underline{\mathbf{R}}}}(t) \mid \nabla_{\nu} \Phi_{\underline{\underline{\mathbf{R}}}}(t)\right\rangle_{\underline{\underline{\mathbf{r}}}}-\frac{\mathbf{A}_{\nu}^{2}(\underline{\underline{\mathbf{R}}}, t)}{2 M_{\nu}}\right),
$$

with the second term on the RHS obtained from the action of the electron-nuclear coupling operator in Eq. 10 ) on the electronic wave-function, and

$$
\epsilon_{G D}(\underline{\underline{\mathbf{R}}}, t)=\left\langle\Phi_{\underline{\underline{\mathbf{R}}}}(t)\left|-i \hbar \partial_{t}\right| \Phi_{\underline{\underline{\mathbf{R}}}}(t)\right\rangle_{\underline{\underline{\mathbf{r}}}} .
$$

The GI part of the TDPES, $\epsilon_{G I}$, is not affected by the gauge transformation (6). The GD part, on the other hand, depends on the choice of the gauge. They both have important features [18] that will be discussed and analyzed in the following section. For this analysis, we will use a representation in terms of the BO electronic states, $\varphi_{\underline{\underline{\mathbf{R}}}}^{(l)}(\underline{\underline{\mathbf{r}}})$, and BOPESs, $\epsilon_{B O}^{(l)}(\underline{\underline{\mathbf{R}}})$, which are the 
eigenstates and eigenvalues of the BO electronic Hamiltonian (2), respectively. If the full wavefunction is expanded in this basis,

$$
\Psi(\underline{\underline{\mathbf{r}}}, \underline{\underline{\mathbf{R}}}, t)=\sum_{l} F_{l}(\underline{\underline{\mathbf{R}}}, t) \varphi_{\underline{\underline{\mathbf{R}}}}^{(l)}(\underline{\underline{\mathbf{r}}})
$$

then the nuclear density may be written as

$$
|\chi(\underline{\underline{\mathbf{R}}}, t)|=\sqrt{\sum_{l}\left|F_{l}(\underline{\underline{\mathbf{R}}}, t)\right|^{2}} .
$$

This identity is obtained by integrating the squared modulus of Eq. 21] over the electronic coordinates with normalized adiabatic states. The exact electronic wave-function may also be expanded in terms of the $\mathrm{BO}$ states,

$$
\Phi_{\underline{\underline{\mathbf{R}}}}(\underline{\underline{\mathbf{r}}}, t)=\sum_{l} C_{l}(\underline{\underline{\mathbf{R}}}, t) \varphi_{\underline{\underline{\mathbf{R}}}}^{(l)}(\underline{\underline{\mathbf{r}}}) .
$$

The expansion coefficients of Eqs. (21) and (23) are related,

$$
F_{l}(\underline{\underline{\mathbf{R}}}, t)=C_{l}(\underline{\underline{\mathbf{R}}}, t) \chi(\underline{\underline{\mathbf{R}}}, t),
$$

by virtue of the factorization (4). The PNC then reads

$$
\sum_{l}\left|C_{l}(\underline{\underline{\mathbf{R}}}, t)\right|^{2}=1, \quad \forall \underline{\underline{\mathbf{R}}}, t
$$

In the cases studied in the following sections, the initial wave-function is the product of a realvalued normalized Gaussian wave-packet, centered at $R_{c}=-4.0 a_{0}$ with variance $\sigma=1 / \sqrt{2.85} a_{0}$ (black line in Fig. 2), and the second BO electronic state, $\varphi_{R}^{(2)}(r)$.

\section{A. Steps in the TDPES in strong non-adiabatic regime}

We first study a case in which the electronic and nuclear motions are strongly coupled. In order to produce that situation, we choose the parameters of the Hamiltonian (14) as $R_{f}=5.0 a_{0}$, $R_{l}=3.1 a_{0}$ and $R_{r}=4.0 a_{0}$ such that the first BOPES, $\epsilon_{B O}^{(1)}$, is strongly coupled to the second

BOPES, $\epsilon_{B O}^{(2)}$, around the avoided crossing at $R_{a c}=-1.90 a_{0}$ and there is a weak coupling to the rest of the surfaces. The four lowest BOPESs for this set of parameters are shown in Fig. 2 (left panel), along with the initial nuclear density. Energies are given in atomic (Hartree) units $\epsilon_{h}$. The same figure (right panel) presents the time-evolution of the populations of the BO states,

$$
\rho_{l}(t)=\int d \underline{\underline{\mathbf{R}}}\left|F_{l}(\underline{\underline{\mathbf{R}}}, t)\right|^{2}
$$



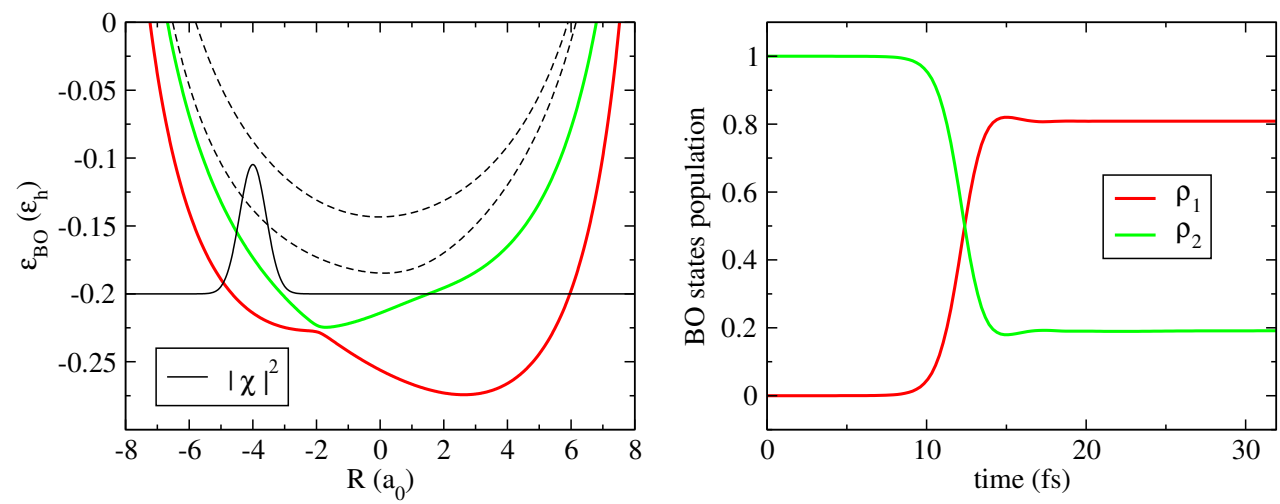

FIG. 2: Left: lowest four BO surfaces, as functions of the nuclear coordinate. The first (red line) and second (green line) surfaces will be considered in the actual calculations that follow, the third and forth (dashed black lines) are shown for reference. The squared modulus (reduced by ten times and rigidly shifted in order to superimpose it on the energy curves) of the initial nuclear wave-packet is also shown (black line). Right: populations of the BO states along the time evolution. The strong non-adiabatic nature of the model is underlined by the population exchange at the crossing of the coupling region.

and underlines the strong non-adiabatic character of the system with the intense population exchange taking place at the passage through the avoided crossing $(t \simeq 12 \mathrm{fs})$.

As recently discussed [18], the GI part of the TDPES (19) shows, in general, two distinct features: (i) in the vicinity of the avoided crossing, as the nuclear wave-packet passes through the region of non-adiabatic coupling between different BOPESs, $\epsilon_{G I}(R, t)$ resembles the diabatic surface that smoothly connects the two adiabatic surfaces; (ii) a bit further away from the avoided crossing, it shows dynamical steps between regions in $R$-space where it is on top of one or the other BOPES. The GD part of the TDPES 201, on the other hand, is a piecewise constant function of the nuclear coordinate. This is illustrated in detail in Fig. 3 that contains the GI part of the TDPES (upper panel), the GD part of the TDPES (middle panel) and the nuclear density together with $\left|F_{1}\right|^{2}$ and $\left|F_{2}\right|^{2}$ (lower panel) for three different snapshots of time. In all the plots, the regions highlighted within the boxes are the regions which we refer to in the following discussion. Outside such regions, the value of the nuclear density drops under the numerical accuracy and the resulting potentials are not meaningful. That is why the TDPES are trimmed. The left panels show, at the initial time-step, (top) the GI part of the TDPES (black dots), with the two lowest BOPESs $\left(\epsilon_{B O}^{(1)}(R)\right.$, dashed red line, and $\epsilon_{B O}^{(2)}(R)$, dashed green line) as reference, (center) the GD part of the exact potential (dark-green dots) and (bottom) the nuclear density (dashed black line) and its components from on the BO states (see Eq. (22)), $\left|F_{1}(R, t)\right|^{2}$ (red line) and $\left|F_{2}(R, t)\right|^{2}$ (green 
line). At time $t=0 \mathrm{fs}$, the electronic wave-function, $\Phi_{R}(r, t)$, coincides with the second adia-
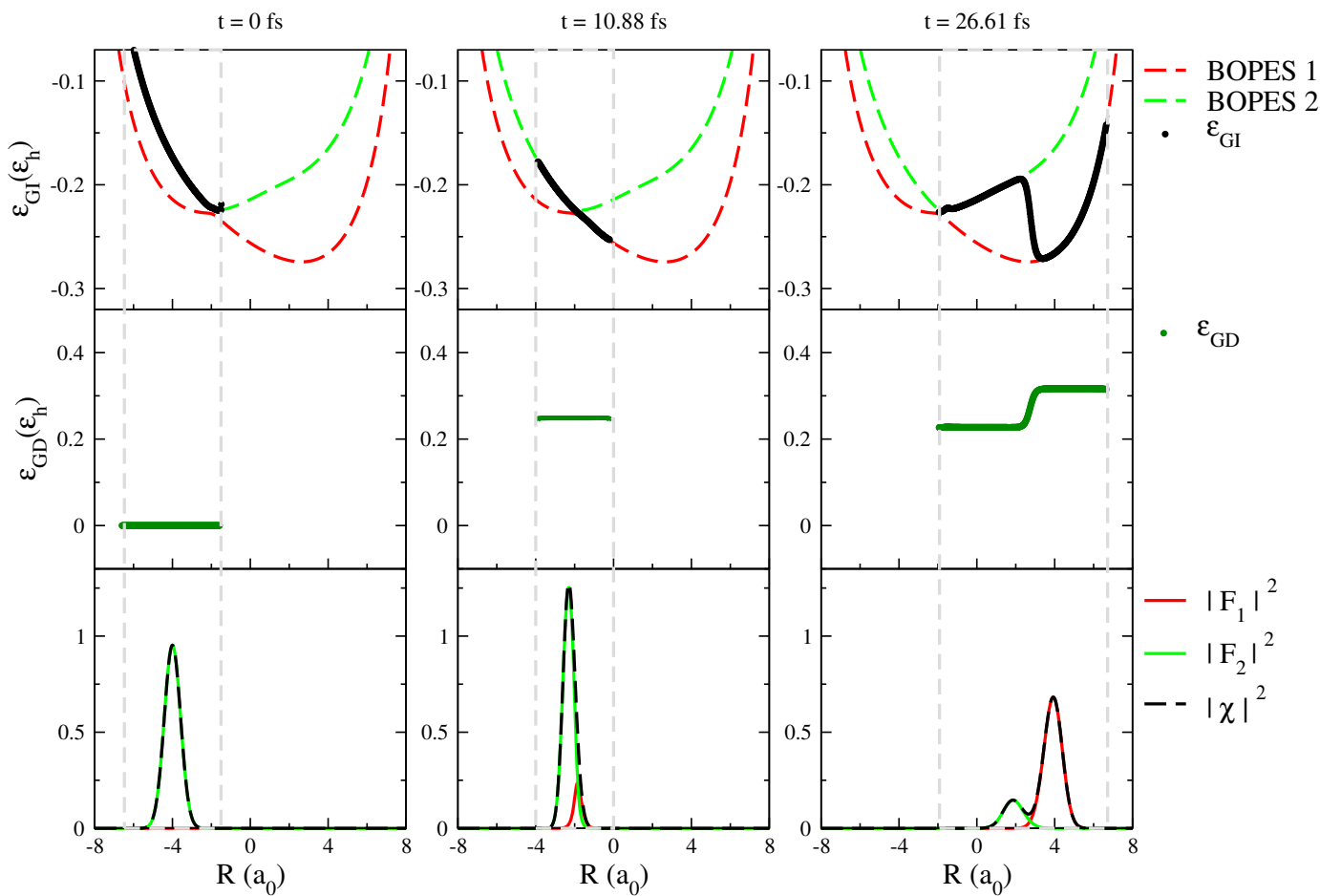

FIG. 3: TDPES and nuclear densities at different time-steps, namely $t=0 \mathrm{fs}, t=10.88 \mathrm{fs}$ and $t=26.61 \mathrm{fs}$. The different panels show: (top) GI part of the TDPES (black dots) and the two lowest BOPESs (first, dashed red line, and second, dashed green line) as reference; (center) the GD part of the TDPES (green dots); (bottom) nuclear density (dashed black line) and $\left|F_{l}(R, t)\right|^{2}(l=1$ red line and $l=2$ green line). The gray boxes define the regions in $R$-space where the energies have been calculated, since the nuclear density is (numerically) not zero.

batic state $\varphi_{R}^{(2)}(r)$, therefore the GI component of the TDPES is identical with $\epsilon_{B O}^{(2)}(R)$, apart from a slight deviation due to the second term in Eq. (19). This is easily confirmed by the expression of $\epsilon_{G I}(R, t)$ in terms of the $\mathrm{BO}$ states and energies

$$
\begin{aligned}
\epsilon_{G I}(R, t) & =\sum_{l}\left|C_{l}(R, t)\right|^{2} \epsilon_{B O}^{(l)}(R)+\frac{\hbar^{2}}{2 M}\left[\sum_{l, k} C_{l}^{*}(R, t) C_{k}(R, t) d_{l k}^{(2)}(R)\right. \\
& \left.\sum_{l, k}\left(C_{l}^{* \prime}(R, t) C_{k}(R, t)-C_{l}^{*}(R, t) C_{k}^{\prime}(R, t)\right) d_{l k}^{(1)}(R)+\sum_{l}\left|C_{l}^{\prime}(R, t)\right|^{2}\right],
\end{aligned}
$$

where we use the prime to indicate the spatial derivative of the coefficients and we introduced the non-adiabatic couplings

$$
\begin{aligned}
& d_{l k}^{(1)}(R)=\left\langle\varphi_{R}^{(l)} \mid \nabla_{R} \varphi_{R}^{(k)}\right\rangle_{r}=-d_{k l}^{(1)^{*}}(R) \\
& d_{l k}^{(2)}(R)=\left\langle\nabla_{R} \varphi_{R}^{(l)} \mid \nabla_{R} \varphi_{R}^{(k)}\right\rangle_{r}=d_{k l}^{(2)^{*}}(R) .
\end{aligned}
$$


The leading term in Eq. 27) is the average of the BOPESs weighted by $\left|C_{l}(R, t)\right|^{2}$, since the second term is $\mathcal{O}\left(M^{-1}\right)$. The GD component of the TDPES in Eq. 20), in terms of the BO states, becomes

$$
\epsilon_{G D}(R, t)=\sum_{l}\left|C_{l}(R, t)\right|^{2} \dot{\gamma}_{l}(R, t)
$$

where $\dot{\gamma}_{l}(R, t)$ is the time-derivative of the phase of the coefficients $C_{l}(R, t)=e^{\frac{i}{\hbar} \gamma_{l}(R, t)}\left|C_{l}(R, t)\right|$. The nuclear density, along with its components on the BO states from Eq. (22), is presented in the bottom panels of Fig. 3. At the initial time, $|\chi(R, t)|^{2}=\left|F_{2}(R, t)\right|^{2}$.

At $t=10.88 \mathrm{fs}$ in Fig. 3 (central panels), (top) the GI part of the TDPES resembles the diabatic surface [24] that smoothly passes through the avoided crossing. This behavior allows the nuclear density moving on the upper BOPES to be partially "transferred" to the lower state, as the consistent increase of the population of state $\varphi_{R}^{(1)}(r)$ (red curve in the bottom plot in Fig. 3) confirms. In the region highlighted by the dashed box, the GD part of the exact potential is constant, therefore, it does not affect nuclear dynamics.

At later times $(t=26.61 \mathrm{fs}$ shown in the right panels of Fig. 3), when the nuclear wave-packet has split at the avoided crossing, both components of the TDPES present a pronounced stepwise behavior: the GI part follows one or the other BOPES in different regions of $R$-space that are connected by a step, whereas the GD part is stepwise constant, with steps appearing in the same region.

The overall shape of the TDPES, at initial times, is determined by the GI part, as the effect of the GD part is no more than a constant shift. Hence, the TDPES, that drives the nuclear dynamics, behaves like a diabatic surface and "opens" in the direction of the wave-packet's motion in order to facilitate the population exchange between the adiabatic states. After the wave-packet splits at the avoided crossing, in different regions in $R$-space, the TDPES is parallel to one or the other BOPES and a step forms in the transition region. Therefore, the motion of the components $F_{l}(R, t)$ of the nuclear wave-packet is driven by single adiabatic surfaces and not (like, e.g., in Ehrenfest dynamics) by an average electronic potential. This feature is reminiscent of the way the well-known trajectory surface hopping (TSH) scheme [14] deals with the non-adiabatic dynamics. In this approach, the components (in our case identified by the symbol $\left|F_{l}(R, t)\right|^{2}$ ) of the nuclear density on different BO states are represented by bundles of classical trajectories evolving, independently from one another, on different BO surfaces. The ratio of the total number of trajectories occupying, at each time, the surfaces approximates the population $\rho_{l}$ of the corresponding BO state. The success of this method in reproducing non-adiabatic processes becomes clear in the light of the fact that the exact TDPES itself is parallel to different BOPESs in different regions 
along the nuclear coordinate. The usually abrupt transitions between the adiabatic surfaces, i.e., the steps in the exact treatment, are reminiscent to the stochastic jumps between $\mathrm{BO}$ surfaces in TSH.

\section{B. Analysis of the steps}

The behavior of the GI part of the TDPES is mainly determined by the first term in Eq. (27). The steps appear in the region around $R_{0}$, the cross-over of $\left.\mid F_{1}(R, t)\right)\left.\right|^{2}$ and $\left.\mid F_{2}(R, t)\right)\left.\right|^{2}$. In particular, at this point $\left|F_{1}\left(R_{0}, t\right)\right|^{2}=\left|F_{2}\left(R_{0}, t\right)\right|^{2}=|X(t)|$ and, irrespective of this value, the expansion coefficients in the electronic wave-function $\left(23\right.$ have the value $\left|C_{1}\left(R_{0}, t\right)\right|^{2}=\left|C_{2}\left(R_{0}, t\right)\right|^{2}=1 / 2$. This relation holds as consequence of Eq. (24), which can be written as

$$
\left|C_{l}\left(R_{0}, t\right)\right|^{2}=\frac{\left|F_{l}\left(R_{0}, t\right)\right|^{2}}{\left|F_{1}\left(R_{0}, t\right)\right|^{2}+\left|F_{2}\left(R_{0}, t\right)\right|^{2}}=\frac{1}{2} \quad \text { with } \quad l=1,2,
$$

and is clearly shown in Fig. 4 . Here we present, in the upper panel, the GI part (black line) and the GD part (blue line, rigidly shifted along the energy axis) of the exact potential at time $t=26.62 \mathrm{fs}$. The BO surfaces (dashed red and green lines) are also plotted as reference. In the lower panel, we plot the coefficients of the expansions in Eq. 21) (dashed red and green lines) and in Eq. 23. (continuous red and green lines). The continuous black line represents the nuclear density.

The expression of the GI component of the TDPES for a two-state system, from Eq. (27), is

$$
\epsilon_{G I}(R, t) \simeq\left|C_{1}(R, t)\right|^{2} \epsilon_{B O}^{(1)}(R)+\left|C_{2}(R, t)\right|^{2} \epsilon_{B O}^{(2)}(R)
$$

neglecting terms $\mathcal{O}\left(M^{-1}\right)$. If $\left|C_{l}(R, t)\right|^{2}$ is Taylor-expanded around $R_{0}$, up to within the linear deviations,

$$
\begin{aligned}
\left|C_{\frac{1}{2}}(R, t)\right|^{2} & \left.\simeq \frac{\left|F_{\frac{1}{2}}(R, t)\right|^{2}}{|\chi(R, t)|^{2}}\right|_{R_{0}}+\left.\nabla_{R} \frac{\left|F_{\frac{1}{2}}(R, t)\right|^{2}}{|\chi(R, t)|^{2}}\right|_{R_{0}}\left(R-R_{0}\right) \\
& =\frac{1}{2} \pm \frac{\alpha(t)}{2}\left(R-R_{0}\right)
\end{aligned}
$$

one can identify the parameter $\alpha(t)$, defined as

$$
\alpha(t)=\frac{\left(\nabla_{R}\left|F_{1}(R, t)\right|\right)_{R_{0}}-\left(\nabla_{R}\left|F_{2}(R, t)\right|\right)_{R_{0}}}{|X(t)|},
$$

where $\alpha(t)$ is the slope of the coefficients in the step region from which the width of the region can be determined. Using the relation $0 \leq\left|C_{1}(R, t)\right|^{2} \leq 1$, we get

$$
0 \leq \frac{1}{2}+\frac{\alpha(t)}{2}\left(R-R_{0}\right) \leq 1 \quad \text { with } \quad \frac{\Delta R}{2}=\left|R-R_{0}\right| \leq \frac{1}{\alpha(t)} .
$$




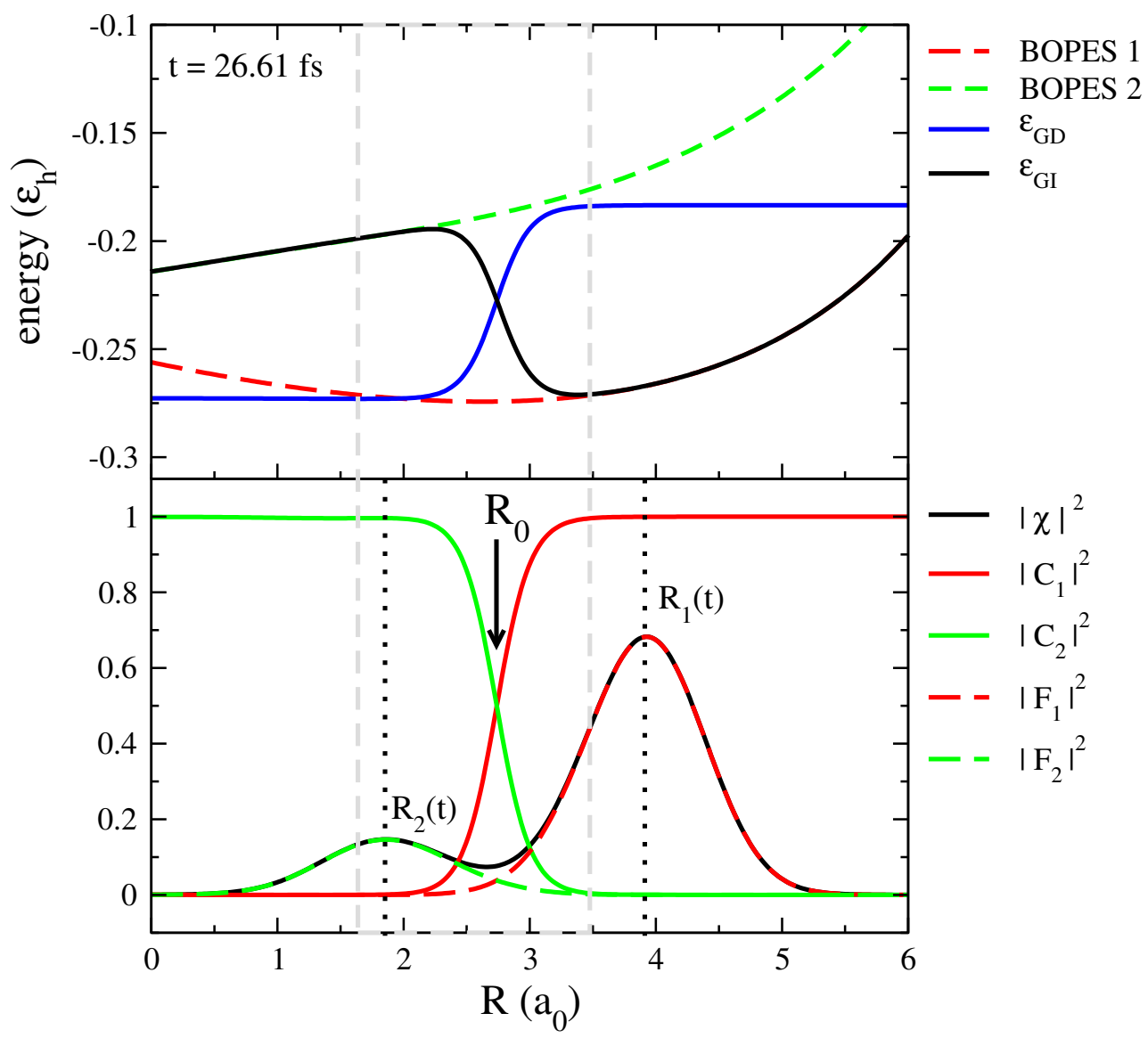

FIG. 4: Top: GI part (black line) and the GD part (blue line, rigidly shifted along the energy axis) of the exact potential at time $t=26.61 \mathrm{fs}$. The first (dashed red) and second (dashed green) BOPESs are shown as reference. Bottom: coefficients $\left|F_{l}(R, t)\right|^{2}$ of the expansion of the full wave-function (Eq. (21)) on the BO states ( $l=1$ dashed red line, $l=2$ dashed green line) and coefficients $\left|C_{l}(R, t)\right|^{2}$ of the expansion of the electronic wave-function ( $l=1$ continuous red line, $l=2$ continuous green line); the black line represents the nuclear density. $R_{0}$ is the position where the coefficients $\left|F_{1}(R, t)\right|^{2}$ and $\left|F_{2}(R, t)\right|^{2}$ have the same value and the dashed box highlights the region of the step.

Therefore, $\Delta R$ is small because the step is steep, as consequence of a large $\alpha(t) . \alpha(t)$ can be large either because $|X(t)|$ is small, i.e., the cross-over is located in a region of small nuclear density, or because the terms in the numerator of Eq. (34) have opposite slopes at $R_{0}$ (this is the case depicted in Fig. (4). Outside the region $\Delta R$, one or the other coefficients $\left|C_{l}(R, t)\right|^{2}$ dominates, thus leading to

$$
\epsilon_{G I}(R, t)=\left\{\begin{array}{l}
\epsilon_{B O}^{(2)}(R), \quad R<R_{0} \\
\epsilon_{B O}^{(1)}(R), \quad R>R_{0} .
\end{array}\right.
$$


The GD part of the TDPES can be analyzed similarly: $\epsilon_{G D}(R, t)$ from Eq. (30) may be written, in terms of the two BO states, as

$$
\epsilon_{G D}(R, t)=\left|C_{1}(R, t)\right|^{2} \dot{\gamma}_{1}(R, t)+\left|C_{2}(R, t)\right|^{2} \dot{\gamma}_{2}(R, t)
$$

and we recall that $\gamma_{l}(R, t)$ is the phase of the coefficient $C_{l}(R, t)$. As in Eq. (36), outside the step region, this part of the potential becomes

$$
\epsilon_{G D}(R, t)= \begin{cases}\dot{\gamma}_{2}(R, t), & R<R_{0} \\ \dot{\gamma}_{1}(R, t), & R>R_{0}\end{cases}
$$

Moreover, Fig. 4 shows that in these regions $\dot{\gamma}_{1}(R, t)$ and $\dot{\gamma}_{2}(R, t)$ are constant functions of $R$. This is a consequence of the gauge we chose. The gauge condition, $A(R, t)=\left\langle\Phi_{R}(t) \mid-i \hbar \nabla_{R} \Phi_{R}(t)\right\rangle_{r}=$ 0 , in terms of the two BO states involved in the dynamics, reads

$$
\begin{array}{r}
0=\sum_{l=1,2}\left|C_{l}(R, t)\right|^{2} \nabla_{R} \gamma_{l}(R, t)-\frac{i \hbar}{2} \nabla_{R} \sum_{l=1,2}\left|C_{l}(R, t)\right|^{2} \\
-i \hbar \sum_{l, k=1,2} C_{l}^{*}(R, t) C_{k}(R, t) d_{l k}^{(1)}(R) .
\end{array}
$$

However, the second term of the RHS is identically zero, due to the PNC in Eq. 25), and the third term can be neglected, due to the presence of the non-adiabatic couplings, $d_{l k}^{(1)}(R)$, that are small far from the avoided crossing. The gauge condition then states

$$
\left|C_{1}(R, t)\right|^{2} \nabla_{R} \gamma_{1}(R, t)=-\left|C_{2}(R, t)\right|^{2} \nabla_{R} \gamma_{2}(R, t)
$$

or equivalently

$$
\begin{array}{lllll}
\nabla_{R} \gamma_{2}(R, t)=0 & \text { for } \quad R<R_{0} & \text { where } & \left|C_{1}(R, t)\right|^{2}=0 \\
\nabla_{R} \gamma_{1}(R, t)=0 & \text { for } \quad R>R_{0} & \text { where } & \left|C_{2}(R, t)\right|^{2}=0 .
\end{array}
$$

We obtain $\gamma_{l}(R, t)=\Gamma_{l}(t)$, namely the phase of the coefficient $C_{l}(R, t)$ is only a function of time (constant in space) in the region where the squared modulus of the corresponding coefficient is equal to unity. Similarly, $\dot{\gamma}_{l}(R, t)=\dot{\Gamma}_{l}(t)$, as shown in Fig. 4 .

In the step region, around $R_{0}$, the expression of the TDPES can be approximated as

$$
\begin{aligned}
\epsilon(R, t)= & \frac{\epsilon_{B O}^{(1)}(R)+\epsilon_{B O}^{(2)}(R)}{2}+\frac{\dot{\gamma}_{1}(R, t)+\dot{\gamma}_{2}(R, t)}{2} \\
& +\alpha(t)\left[\frac{\epsilon_{B O}^{(1)}(R)-\epsilon_{B O}^{(2)}(R)}{2}+\frac{\dot{\gamma}_{1}(R, t)-\dot{\gamma}_{2}(R, t)}{2}\right]\left(R-R_{0}\right) .
\end{aligned}
$$


The first two terms on the RHS are the average of the BO energies plus the average value of the time-derivative of the phases $\gamma_{1}(R, t)$ and $\gamma_{2}(R, t)$; the terms in square brackets are the energy gaps between the $\mathrm{BO}$ surfaces and between the time-derivative of the phases, which give the contribution proportional to the parameter $\alpha(t)$. From Fig. 4 , we notice that, around $R_{0}$, the slope of $\epsilon_{G D}$ is opposite to the slope of $\epsilon_{G I}$ and this is a general feature in the studied system (in the absence of a time-dependent external field). Therefore, the GD part reduces the height of the steps in the GI part. We will see the effect of this contribution on (classical) nuclear dynamics in the section IV

\section{Steps in the TDPES in weak non-adiabatic regime}

In this section, we study a case of weaker non-adiabatic coupling between the two lowest BO states. In order to make the coupling weaker, we choose the parameters in the Hamiltonian (14) as $L=19.0 a_{0}, R_{f}=3.8 a_{0}, R_{l}=2.0 a_{0}$ and $R_{r}=5.5 a_{0}$. The BO surfaces, along with the evolution of the populations of the BO states, are shown in Fig. 5. The initial conditions for the dynamical
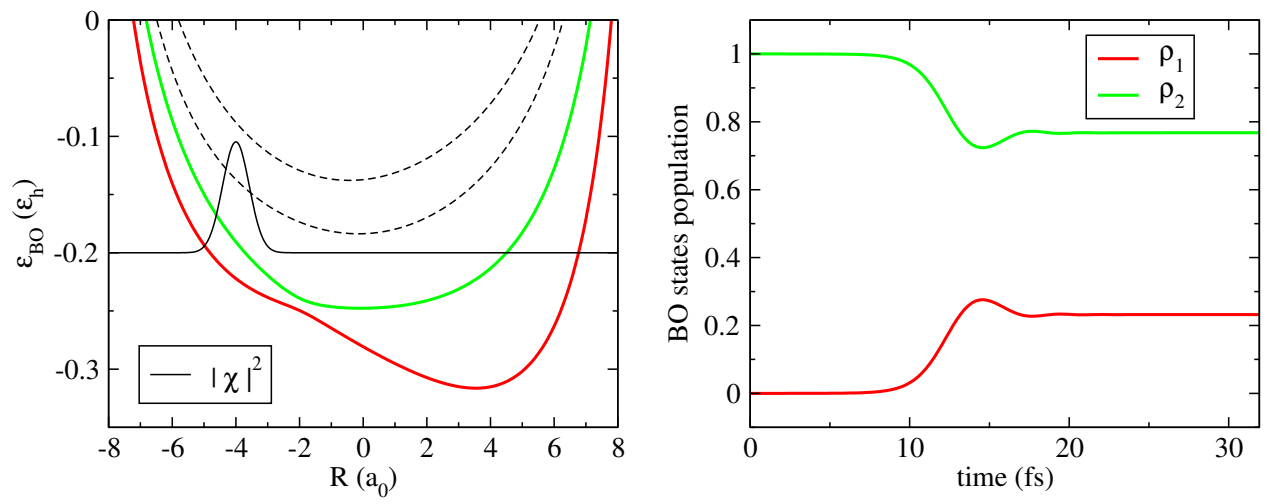

FIG. 5: Same as Fig. 2 but for weaker non-adiabatic coupling between the two lowest BO states.

evolution of this system are the same as in the previous example, however the coupling between the two lowest electronic states is weaker, thus leading to a reduced population exchange, clearly shown in Fig. 5 (right panel). Nonetheless, the process described here shows similarities to the previous case, as can be seen from Fig. 6. The GI part of the TDPES presents again two main features, (i) the diabatization at the avoided crossing, when the nuclear wave-packet crosses the region of relatively strong non-adiabatic coupling and (ii) the steps at the cross-over of $\left|F_{1}(R, t)\right|^{2}$ and $\left|F_{2}(R, t)\right|^{2}$, signature of the splitting of the nuclear density. The GD part is either constant, before the splitting at the avoided crossing, or stepwise constant, with steps appearing in the 

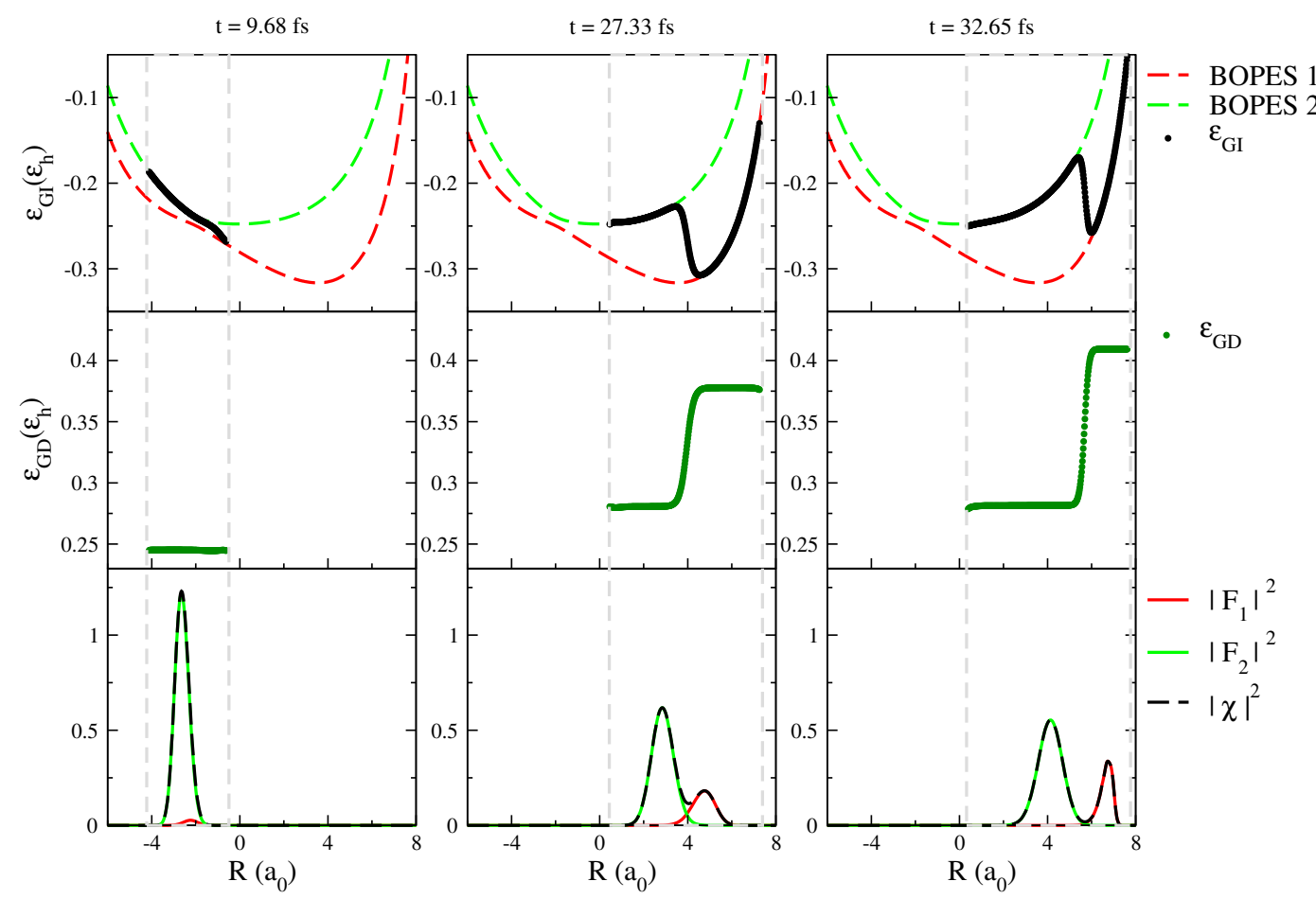

FIG. 6: Same as Fig. 3 but for a weaker non-adiabatic coupling between the two lowest BO states, at timesteps $9.68 f s, 27.33 \mathrm{fs}$ and $32.65 \mathrm{fs}$.

same region as the steps in the GI term, but with opposite slope. At different snapshots of time, i.e., $9.68 f s, 27.33 f s$ and $32.65 f s$, these properties are shown in Fig. 6 , along with the nuclear density and its components on the BO states. The notation used in the figures is the same as in Fig. 3 .

A slightly different behavior from the situation of strong non-adiabatic coupling can be identified in $\epsilon_{G I}(R, t)$ before the passage through the avoided crossing. As the nuclear wave-packet approaches the avoided crossing, the GI part of the TDPES "opens" towards the direction of motion, resembling the diabatic surface that connects the BO surfaces through the avoided crossing. This is clearly shown in Fig. 7 (left) at time $t=9.68 \mathrm{fs}$ for the strongly coupled system. In the case of weaker non-adiabatic coupling, $\epsilon_{G I}(R, t)$, at the avoided crossing, lies between the BO surfaces, as shown in Fig.7(right). Therefore, the diabatization feature strictly depends on the strength of the non-adiabatic coupling and, in general, can be viewed as a transient configuration of the GI part of the TDPES before the formation of the steps. 

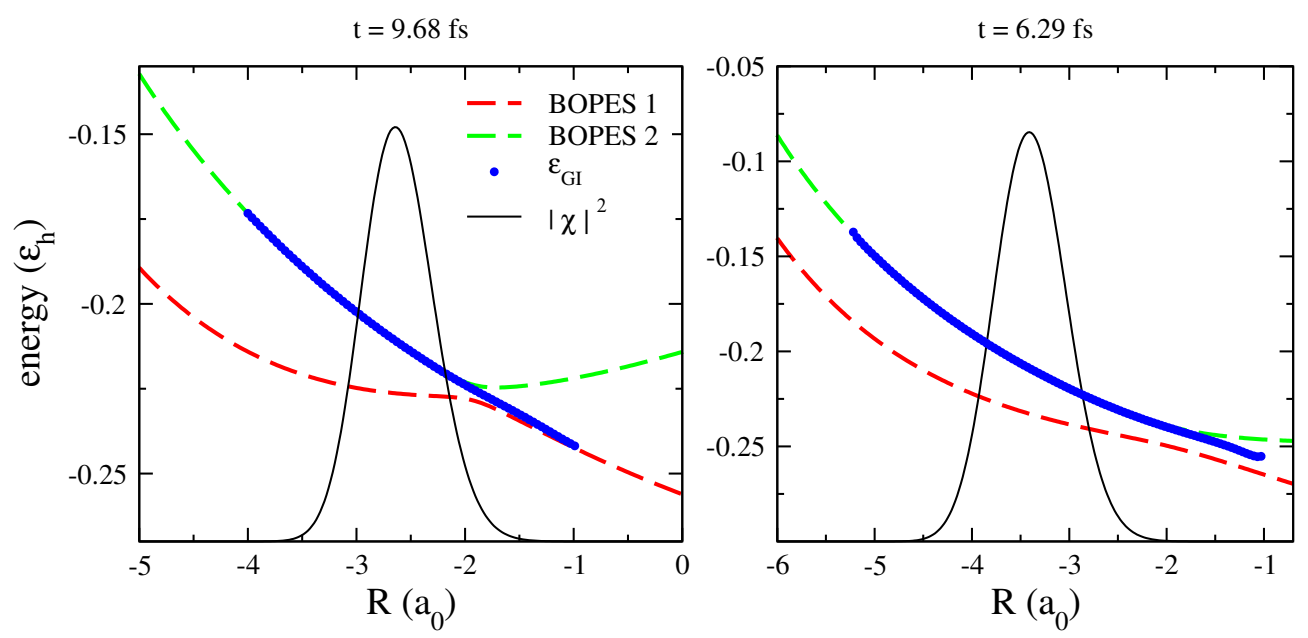

FIG. 7: Diabatization feature of $\epsilon_{G I}(R, t)$ (blue dots) for the two model systems (left panel, strong coupling at $t=9.68 \mathrm{fs}$, and right panel, weak coupling at $t=6.29 \mathrm{fs}$ ) presented here. The dashed lines represent the BO surfaces $\left(\epsilon_{B O}^{(1)}(R)\right.$ red line and $\epsilon_{B O}^{(2)}(R)$ green line) and the continuous black line represents the nuclear density (reduced by a factor 10 and rigidly shifted along the $y$-axis).

\section{CLASSICAL DYNAMICS ON PESS}

In section III. we have addressed some of the generic features of the TDPES that governs the nuclear dynamics in the presence of non-adiabatic electronic transitions. As discussed before, some of these features, in particular the step that bridges between the two parts of the TDPES that are parallel to the BOPESs, are reminiscent of the jumping between the BOPESs in TSH methods [14]. These algorithms are based on the mixed quantum-classical treatment of the electronic and nuclear dynamics using stochastic jumps between BO surfaces. Therefore, an ensemble of classical trajectories with different initial conditions is needed to achieve statistically reasonable outcomes. On the other hand, the TDPES is the exact time-dependent potential that governs the nuclear dynamics (in general together with the vector potential) and contains the back-reaction resulting from the exact coupling to the electronic subsystem. This brings us to investigate how the TDPES drives the classical dynamics of point-like nuclei.

In order to understand how the generic features of the TDPES affect the classical nuclear dynamics, we have employed the surfaces presented in section (III) to calculate the forces acting on the nuclear degree of freedom. We compare the resulting dynamics using the forces that are calculated from the gradient of the TDPES and from the gradient of its GI part. The classical propagation starts at the initial position $R_{c}=-4.0 a_{0}$ with zero initial momentum. Here, we use the 
velocity-Verlet algorithm to integrate Hamilton's equations,

$$
\left\{\begin{array}{l}
\dot{R}=\frac{P}{M} \\
\dot{P}=-\nabla_{R} \epsilon(R) \text { or }-\nabla_{R} \epsilon_{G I}(R),
\end{array}\right.
$$

using the same time-steps as in the quantum propagation $\left(\delta t=2.4 \times 10^{-3} f s\right)$. In Fig. 8 (upper panels) we present the evolution of the classical position compared to the average nuclear position from the quantum calculation, for strong and weak coupling. In both cases, a single trajectory, evolving on the exact surface (blue lines in Fig. 8), is able to reproduce the mean nuclear path (dashed black lines) fairly well. A slight deviation from the quantum results happens only towards the end of the simulated trajectories. When the classical forces are calculated from the GI part of the TDPES, the corresponding classical trajectory in the strong coupling case, does not show a large deviation from the exact calculation. However, in the weak coupling case, after $20 \mathrm{fs}$, the classical trajectory deviates considerably from the quantum mean path. This behavior is also confirmed by the pronounced increase of the velocity of the classical particle moving on $\epsilon_{G I}$, shown in Fig. 8 (lower panels).

We now have a closer look at the classical dynamics and try to find out the source of the deviations, especially in the weaker coupling case. Fig. 9 shows the classical positions calculated from the full TDPES (blue dots) and the GI part of it (orange dots) together with the corresponding potentials and the exact nuclear densities at the times indicated in the plots. It can be seen in the figure that the classical particle evolving on the GI part of the potential, in the case of weaker coupling, at the moment of the step formation feels an intense force, as its position is exactly in the region of the step (see $t=23.71 \mathrm{fs}$ in Fig. 9). This happens also in the case of the strong coupling (see the blue line referring to the velocity in Fig. 8, left plot), to a lesser extent and the velocity of the classical particle does not show a strong peak. The evolution of the classical particle on the GI part, in the case of the strong coupling, shows that the step forms in the direction of larger nuclear density (see plot at $t=22.25 \mathrm{fs}$ ), hence, the classical particle correctly follows the step and its position is approximately the mean nuclear position. However, in the case of weaker coupling, the step forms in the direction of smaller nuclear density and the classical particle can not move "up the hill" to follow the nuclear mean path, leading to a large deviation of the classical position from the quantum mean value. The intense force felt by the classical particle drives it to an unphysical region, where the nuclear density is very small. The presence of the GD part of the TDPES is responsible for the decrease (or even the inversion) of the "energy gap" in the GI part, 


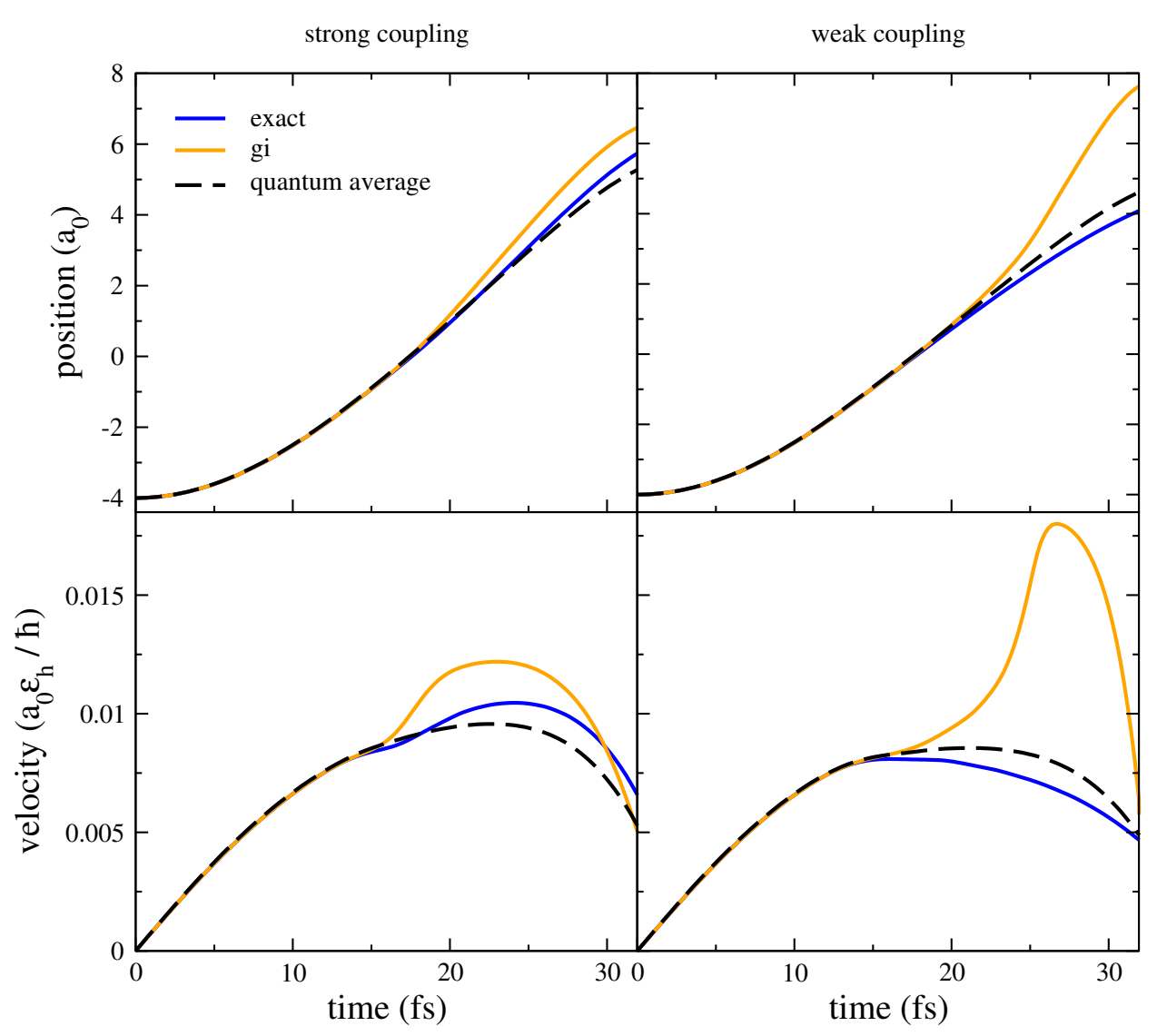

FIG. 8: Classical position (upper panels) and velocity (lower panels) and mean nuclear position and velocity as functions of time for the systems in the presence of strong non-adiabatic coupling (left) and of weak non-adiabatic coupling (right). The dashed black line represents the average nuclear values from quantum calculation, the blue and orange lines are the positions and velocities of the classical particle when it evolves on the exact potential and on the GI part of the potential, respectively.

thus producing a better agreement between classical and quantum results.

From comparing the classical and quantum dynamics shown in Fig. 9, we observe that in the strong coupling case (upper panel), at $t=4.84 \mathrm{fs}$ and at $t=11.37 \mathrm{fs}$, the nuclear wave-packet has not yet crossed the avoided crossing, thus the GD part of the TDPES is a constant. Therefore, the classical force calculated from the TDPES is identical with the one calculated from its GI part. At these times, the classical positions of the nuclei evolving on the GI part of the potential (orange dots in the figure) and on the full TDPES (blue dots) coincide with the mean position of the nuclear wave-packet (black arrows). On the other hand, in the weaker coupling case (lower panels), a similar behavior is seen only before the wave-packet splitting, at $t=7.26 \mathrm{fs}$ and $t=12.09 \mathrm{fs}$. At later times, namely $t=22.25 \mathrm{fs}$ for the strong coupling case and $t=23.71 \mathrm{fs}$ for the weaker 

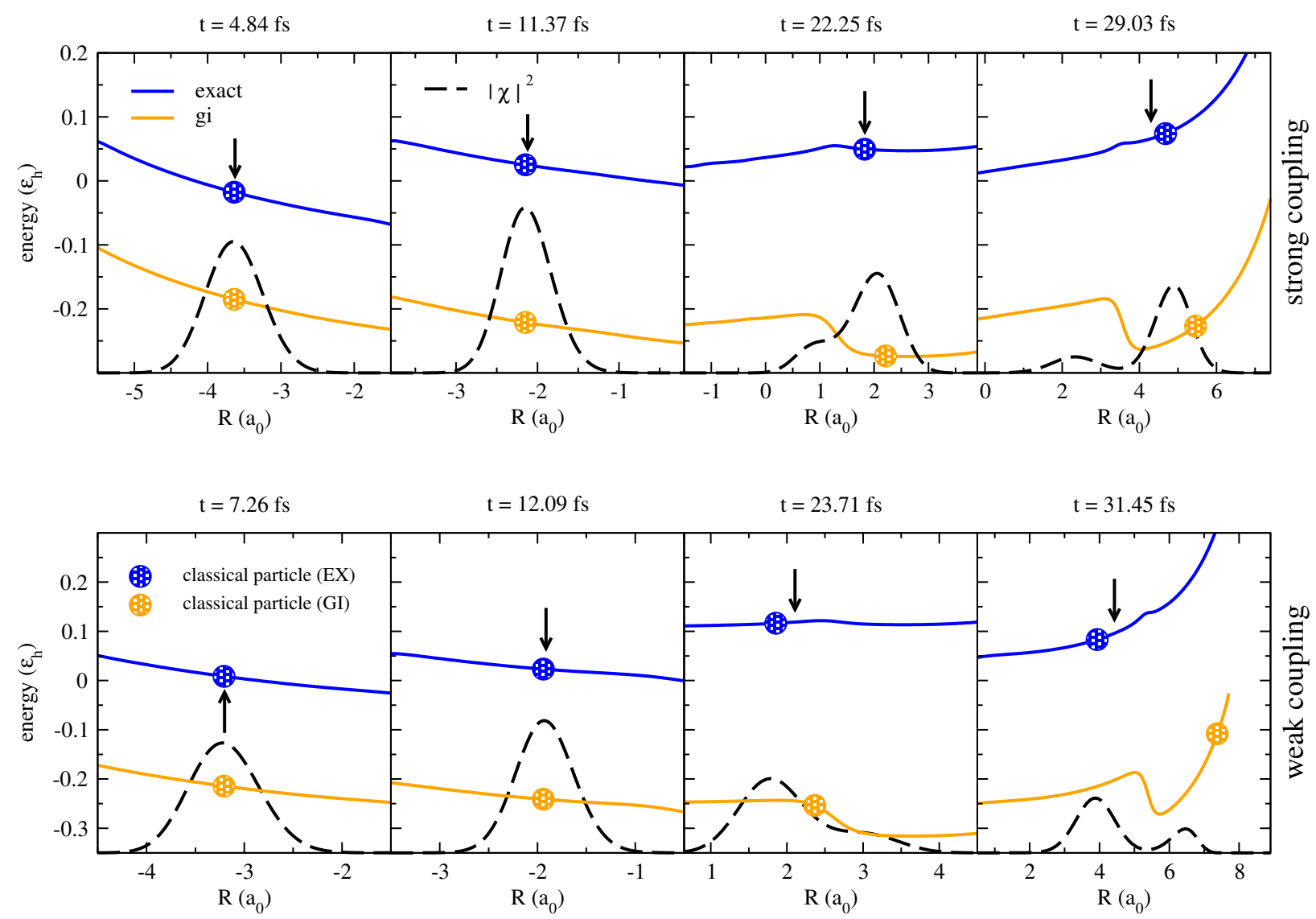

FIG. 9: Upper panels: strong coupling results. Lower panels: weak coupling results. The figure shows classical positions (dots) at different times, as indicated in the plots, with the corresponding potentials, $\epsilon_{G I}(R, t)$ (orange lines) and $\epsilon(R, t)$ (blue lines). The nuclear density (dashed black line) is plotted as reference, along with the mean position (black arrows).

coupling case, the steps develop in $\epsilon_{G I}$ and the classical particle evolving on this potential follows the direction in which the step is forming: in the case of strong coupling, this region coincides with the region associated with larger nuclear density, whereas this is not the case for the weaker coupling case. As discussed above, this feature explains why the positions of the particles on $\epsilon$ and on $\epsilon_{G I}$, for the system in the presence of strong non-adiabatic coupling, are close to each other also at later times $(t=29.03 \mathrm{fs}$ in Fig. 9), whereas they deviate in the weaker coupling regime as clearly shown in the figure at time $t=31.45 \mathrm{fs}$.

The results presented in this section offer interesting insights into possible ways of modeling non-adiabatic processes, within a mixed quantum-classical treatment. On one hand, the gradient of the GI part of the exact potential is the force that drives the classical nuclear motion and we have shown that such force is "adiabatic" in the sense that, far from the step, it is produced by a single 
BOPES. On the other hand, the GD part does not affect such force, but contributes in diminishing the energy separation between the two sides of the step. This energy barrier almost disappears in the full TDPES, but the difference in slopes indeed persists. If a gauge is chosen such that $\epsilon_{G D}(R, t) \equiv 0$, the non-zero vector potential compensates the effect of the energy step in the GI part of the TDPES by adding a kinetic energy contribution (the vector potential appears in the kinetic term of the nuclear Hamiltonian in Eq. (8)). Such contribution would energetically favor the transfer of classical point-particles from one side of the step to the other. Once again, the comparison with TSH is inevitable: in the latter, different adiabatic surfaces are energetically accessible by the classical nuclei because of the stochastic jumps and the subsequent momentum rescaling (in order to impose energy conservation); in the scheme based on the exact TDPES, depending on the gauge, either the GD part of the potential is responsible for bringing "energetically closer" different BOPES or the vector potential gives the necessary kinetic energy contribution. So far, we have described where the steps appear, how they form and how they affect nuclear motion. From these observations, we expect that rigorous mixed quantum-classical schemes for dealing with non-adiabatic processes can be deduced in a systematic way from the classical forces associated with the exact TDPES and the exact vector potential.

\section{EHRENFEST THEOREM FOR THE NUCLEAR WAVE-FUNCTION}

In section IV, we studied the classical nuclear dynamics on the TDPES. However, we did not provide any argument on how that study can be associated with a classical limit of the nuclear motion that is able to, approximately, reproduce the expectation values of the nuclear position and momentum of the complete electron-nuclear system. Here, using the Ehrenfest theorem, we show how the nuclear position and momentum calculated from Eq. 44 can be linked to the expectation values of the nuclear position and momentum of the complete electron-nuclear system.

The Ehrenfest theorem [15] relates the time-derivative of the expectation value of a quantummechanical operator $\hat{O}$ to the expectation value of the commutator of that operator with the Hamiltonian, i.e.

$$
\frac{d}{d t}\langle\hat{O}(t)\rangle=\frac{1}{i \hbar}\langle[\hat{O}(t), \hat{H}]\rangle+\left\langle\partial_{t} \hat{O}(t)\right\rangle
$$

The second term on the RHS refers to the explicit time-dependence of $\hat{O}$. In particular, the theorem

leads to the classical-like equations of motion for the mean value of position and momentum operators. For a system of electrons and nuclei, described by the Hamiltonian in Eq. (1) and the 
wave-function $\Psi(\underline{\underline{\mathbf{r}}}, \underline{\underline{\mathbf{R}}}, t)$, the mean values of the $\nu$-th nuclear position $\hat{\mathbf{R}}_{\nu}$ and momentum $\hat{\mathbf{P}}_{\nu}$ operators evolve according to the classical Hamilton's equations

$$
\begin{aligned}
& \frac{d}{d t}\left\langle\hat{\mathbf{R}}_{\nu}\right\rangle_{\Psi}=\frac{1}{i \hbar}\left\langle\left[\hat{\mathbf{R}}_{\nu}, \hat{H}(\underline{\underline{\mathbf{r}}}, \underline{\underline{\mathbf{R}}})\right]\right\rangle_{\Psi}=\frac{\left\langle\hat{\mathbf{P}}_{\nu}\right\rangle_{\Psi}}{M_{\nu}} \\
& \frac{d}{d t}\left\langle\hat{\mathbf{P}}_{\nu}\right\rangle_{\Psi}=\frac{1}{i \hbar}\left\langle\left[\hat{\mathbf{P}}_{\nu}, \hat{H}(\underline{\underline{\mathbf{r}}}, \underline{\underline{\mathbf{R}}})\right]\right\rangle_{\Psi}=\left\langle-\nabla_{\nu}\left(\hat{V}_{e n}(\underline{\underline{\mathbf{r}}}, \underline{\underline{\mathbf{R}}})+\hat{W}_{n n}(\underline{\underline{\mathbf{R}}})\right)\right\rangle_{\Psi} \cdot
\end{aligned}
$$

Here, the operators do not depend explicitly on time and we indicate the integration over the full wave-function (electronic and nuclear coordinates) by $\langle\cdot\rangle_{\Psi}$. On the other hand, the nuclear equation (8) is a Schrödinger equation that contains a time-dependent vector potential and a timedependent scalar potential. Therefore, the Ehrenfest theorem for the nuclear subsystem reads

$$
\begin{aligned}
\frac{d}{d t}\left\langle\hat{\mathbf{R}}_{\nu}\right\rangle_{\chi} & =\frac{1}{i \hbar}\left\langle\left[\hat{\mathbf{R}}_{\nu}, \hat{H}_{n}(\underline{\underline{\mathbf{R}}})\right]\right\rangle_{\chi} \\
\frac{d}{d t}\left\langle\hat{\widetilde{\mathbf{P}}}_{\nu}\right\rangle_{\chi} & =\frac{1}{i \hbar}\left\langle\left[\hat{\widetilde{\mathbf{P}}}_{\nu}, \hat{H}_{n}(\underline{\underline{\mathbf{R}}})\right]\right\rangle_{\chi}+\left\langle\partial_{t} \mathbf{A}_{\nu}(\underline{\underline{\mathbf{R}}}, t)\right\rangle_{\chi}
\end{aligned}
$$

where [20]

$$
\hat{\widetilde{\mathbf{P}}}_{\nu}=-i \hbar \nabla_{\nu}+\mathbf{A}_{\nu}(\underline{\underline{\mathbf{R}}}, t)
$$

is the expression of the nuclear canonical momentum operator in position representation, and

$$
\hat{H}_{n}(\underline{\underline{\mathbf{R}}})=\sum_{\nu=1}^{N_{n}} \frac{\left[-i \hbar \nabla_{\nu}+\mathbf{A}_{\nu}(\underline{\underline{\mathbf{R}}}, t)\right]^{2}}{2 M_{\nu}}+\epsilon(\underline{\underline{\mathbf{R}}}, t)
$$

is the nuclear Hamiltonian from Eq. (8). Note that the average operation is performed only on the nuclear wave-function as indicated by $\langle\cdot\rangle_{\chi}$. An explicit time-dependence appears in the expression of the momentum operator, due to the presence of the vector potential. This dependence is accounted for in the second term on the RHS of Eq. (67). While Eq. (66) is easily obtained from Eq. (64) by performing the integration over the electronic part of full wave-function, Eq. (67) is more involved and will be proved as follows. We rewrite LHS of Eq. 65) as

$$
\begin{aligned}
\frac{d}{d t}\left\langle\hat{\mathbf{P}}_{\nu}\right\rangle_{\Psi}= & \int d \underline{\underline{\mathbf{r}}} d \underline{\underline{\mathbf{R}}}\left[\Phi_{\underline{\underline{\mathbf{R}}}}^{*}(\underline{\underline{\mathbf{r}}}, t) \partial_{t} \chi^{*}(\underline{\underline{\mathbf{R}}}, t)+\chi^{*}(\underline{\underline{\mathbf{R}}}, t) \partial_{t} \Phi_{\underline{\underline{\mathbf{R}}}}^{*}(\underline{\underline{\mathbf{r}}}, t)\right] \hat{\mathbf{P}}_{\nu} \chi(\underline{\underline{\mathbf{R}}}, t) \Phi_{\underline{\underline{\mathbf{R}}}}(\underline{\underline{\mathbf{r}}}, t) \\
& \left.+\int d \underline{\underline{\mathbf{r}}} d \underline{\underline{\mathbf{R}}} \chi^{*}(\underline{\underline{\mathbf{R}}}, t) \Phi_{\underline{\underline{\mathbf{R}}}}^{*} \underline{\underline{\mathbf{r}}}, t\right) \hat{\mathbf{P}}_{\nu}\left[\Phi_{\underline{\underline{\mathbf{R}}}}(\underline{\underline{\mathbf{r}}}, t) \partial_{t} \chi(\underline{\underline{\mathbf{R}}}, t)+\chi(\underline{\underline{\mathbf{R}}}, t) \partial_{t} \Phi_{\underline{\underline{\mathbf{R}}}}(\underline{\underline{\mathbf{r}}}, t)\right]
\end{aligned}
$$

$\hat{\mathbf{P}}_{\nu}$ being a differential operator in position representation, its action on the factorized wavefunction is

$$
\hat{\mathbf{P}}_{\nu} \chi(\underline{\underline{\mathbf{R}}}, t) \Phi_{\underline{\underline{\mathbf{R}}}}(\underline{\underline{\mathbf{r}}}, t)=\left(\hat{\mathbf{P}}_{\nu} \chi(\underline{\underline{\mathbf{R}}}, t)\right) \Phi_{\underline{\underline{\mathbf{R}}}}(\underline{\underline{\mathbf{r}}}, t)+\chi(\underline{\underline{\mathbf{R}}}, t)\left(\hat{\mathbf{P}}_{\nu} \Phi_{\underline{\underline{\mathbf{R}}}}(\underline{\underline{\mathbf{r}}}, t)\right)
$$


Then we use the nuclear equation (8) for

$$
\partial_{t} \chi(\underline{\underline{\mathbf{R}}}, t)=\frac{1}{i \hbar} \hat{H}_{n}(\underline{\underline{\mathbf{R}}}) \chi(\underline{\underline{\mathbf{R}}}, t)
$$

and its complex-conjugated $\left(\hat{H}_{n}(\underline{\underline{\mathbf{R}}})\right.$ is hermitian), the definition of the (real) vector potential

$$
\left.\mathbf{A}_{\nu}(\underline{\underline{\mathbf{R}}}, t)=\int d \underline{\underline{\mathbf{r}}} \Phi_{\underline{\underline{\mathbf{R}}}}^{*} \underline{\underline{\mathbf{r}}}, t\right) \hat{\mathbf{P}}_{\nu} \Phi_{\underline{\underline{\mathbf{R}}}}(\underline{\underline{\mathbf{r}}}, t)
$$

and the PNC, to derive

$$
\begin{aligned}
& \frac{d}{d t}\left\langle\hat{\mathbf{P}}_{\nu}\right\rangle_{\Psi}=\frac{1}{i \hbar} \int d \underline{\underline{\mathbf{R}}} \chi^{*}(\underline{\underline{\mathbf{R}}}, t)\left(\hat{\tilde{\mathbf{P}}}_{\nu} \hat{H}_{n}(\underline{\underline{\mathbf{R}}})-\hat{H}_{n}(\underline{\underline{\mathbf{R}}}) \hat{\tilde{\mathbf{P}}}_{\nu}\right) \chi(\underline{\underline{\mathbf{R}}}, t) \\
& \left.\quad+\int d \underline{\underline{\mathbf{R}}}|\chi(\underline{\underline{\mathbf{R}}}, t)|^{2} \int d \underline{\underline{\mathbf{r}}}\left[\left(\partial_{t} \Phi_{\underline{\underline{\mathbf{R}}}}^{*} \underline{\underline{\mathbf{r}}}, t\right)\right) \hat{\mathbf{P}}_{\nu} \Phi_{\underline{\underline{\mathbf{R}}}}(\underline{\underline{\mathbf{r}}}, t)+\Phi_{\underline{\underline{\mathbf{R}}}}^{*} \underline{\underline{\mathbf{r}}, t)} \hat{\mathbf{P}}_{\nu} \partial_{t} \Phi_{\underline{\underline{\mathbf{R}}}}(\underline{\underline{\mathbf{r}}}, t)\right]
\end{aligned}
$$

with $\hat{\tilde{\mathbf{P}}}_{\nu}=\hat{\mathbf{P}}_{\nu}+\mathbf{A}_{\nu}(\underline{\underline{\mathbf{R}}}, t)$. Using the relation

$$
\left.\left(\partial_{t} \Phi_{\underline{\underline{\mathbf{R}}}}^{*} \underline{\underline{\mathbf{r}}}, t\right)\right) \hat{\mathbf{P}}_{\nu} \Phi_{\underline{\mathbf{R}}}(\underline{\underline{\mathbf{r}}}, t)=\partial_{t}\left(\Phi_{\underline{\underline{\mathbf{R}}}}^{*}(\underline{\underline{\mathbf{r}}}, t) \hat{\mathbf{P}}_{\nu} \Phi_{\underline{\mathbf{R}}}(\underline{\underline{\mathbf{r}}}, t)\right)-\Phi_{\underline{\underline{\mathbf{R}}}}^{*}(\underline{\underline{\mathbf{r}}}, t) \hat{\mathbf{P}}_{\nu} \partial_{t} \Phi_{\underline{\mathbf{R}}}(\underline{\underline{\mathbf{r}}}, t)
$$

for the term in the square brackets, leads to

$$
\frac{d}{d t}\left\langle\hat{\mathbf{P}}_{\nu}\right\rangle_{\Psi}=\int d \underline{\underline{\mathbf{R}}} \chi^{*}(\underline{\underline{\mathbf{R}}}, t)\left(\frac{1}{i \hbar}\left[\hat{\tilde{\mathbf{P}}}_{\nu}, \hat{H}_{n}(\underline{\underline{\mathbf{R}}})\right]+\partial_{t} \mathbf{A}_{\nu}(\underline{\underline{\mathbf{R}}}, t)\right) \chi(\underline{\underline{\mathbf{R}}}, t)
$$

recovering the term on the RHS of Eq. [67). A similar procedure [20] yields the relation

$$
\begin{aligned}
\left\langle\hat{\mathbf{P}}_{\nu}\right\rangle_{\Psi} & \left.=\int d \underline{\underline{\mathbf{r}}} d \underline{\underline{\mathbf{R}}} \Phi_{\underline{\underline{\mathbf{R}}}}^{*}(\underline{\underline{\mathbf{r}}}, t) \chi^{*} \underline{\underline{\mathbf{R}}}, t\right)\left[\left(\hat{\mathbf{P}}_{\nu} \chi(\underline{\underline{\mathbf{R}}}, t)\right) \Phi_{\underline{\underline{\mathbf{R}}}}(\underline{\underline{\mathbf{r}}}, t)+\chi(\underline{\underline{\mathbf{R}}}, t) \hat{\mathbf{P}}_{\nu} \Phi_{\underline{\underline{\mathbf{R}}}}(\underline{\underline{\mathbf{r}}}, t)\right] \\
& \left.=\int d \underline{\underline{\mathbf{R}}} \chi^{*} \underline{\underline{\mathbf{R}}}, t\right)\left[\hat{\mathbf{P}}_{\nu}+\mathbf{A}_{\nu}(\underline{\underline{\mathbf{R}}}, t)\right] \chi(\underline{\underline{\mathbf{R}}}, t)=\left\langle\hat{\widetilde{\mathbf{P}}}_{\nu}\right\rangle_{\chi},
\end{aligned}
$$

which proves the identity of the LHSs of Eqs. (65) and (67).

We have proved the Ehrenfest theorem for the nuclear wave-function and nuclear Hamiltonian, deriving exact relations for the evolution of the mean values of nuclear position and momentum operators over the complete system. This outcome is consistent with the interpretation of $\chi(\underline{\underline{\mathbf{R}}}, t)$ as the proper nuclear wave-function that reproduces the nuclear density and current density of the complete system (see the discussion in section II).

In the one-dimensional system studied here, the gauge is chosen such that $A(R, t)=0$, therefore, the Ehrenfest equations become

$$
\begin{aligned}
\frac{d}{d t}\langle\hat{R}\rangle_{\chi} & =\frac{1}{i \hbar}\left\langle\left[\hat{R}, \hat{H}_{n}\right]\right\rangle_{\chi}=\frac{\langle\hat{P}\rangle_{\chi}}{M} \\
\frac{d}{d t}\langle\hat{P}\rangle_{\chi} & =\frac{1}{i \hbar}\left\langle\left[\hat{P}, \hat{H}_{n}\right]\right\rangle_{\chi}=\left\langle-\nabla_{R} \epsilon(R, t)\right\rangle_{\chi}
\end{aligned}
$$


where the mean force generating the classical-like evolution is determined as the expectation value, on the nuclear wave-function, of the gradient of the TDPES. If we replace the nuclear wavefunction in Eqs. (78) and (79) by a delta-function centered at the classical position, we get Eqs. (44) that was used in section IV] to generate classical dynamics on the exact PES. That is why the classical nuclear dynamics on the TDPES could actually approximate the mean nuclear position and momentum.

We have numerically simulated classical dynamics under the following equations of motion

$$
\left\{\begin{array}{l}
\dot{R}=\frac{P}{M} \\
\dot{P}=\left\langle-\nabla_{R} \epsilon(R, t)\right\rangle_{\chi},
\end{array}\right.
$$

where $\epsilon(R, t)$ is obtained from the solution of the TDSE with Hamiltonian (14), for both sets of parameters producing strong and weak non-adiabatic coupling between the two lowest BO surfaces. The initial conditions for the classical evolution are exactly the initial mean position and mean velocity of the quantum particle. The results are shown in Fig. 11, where we plot the mean
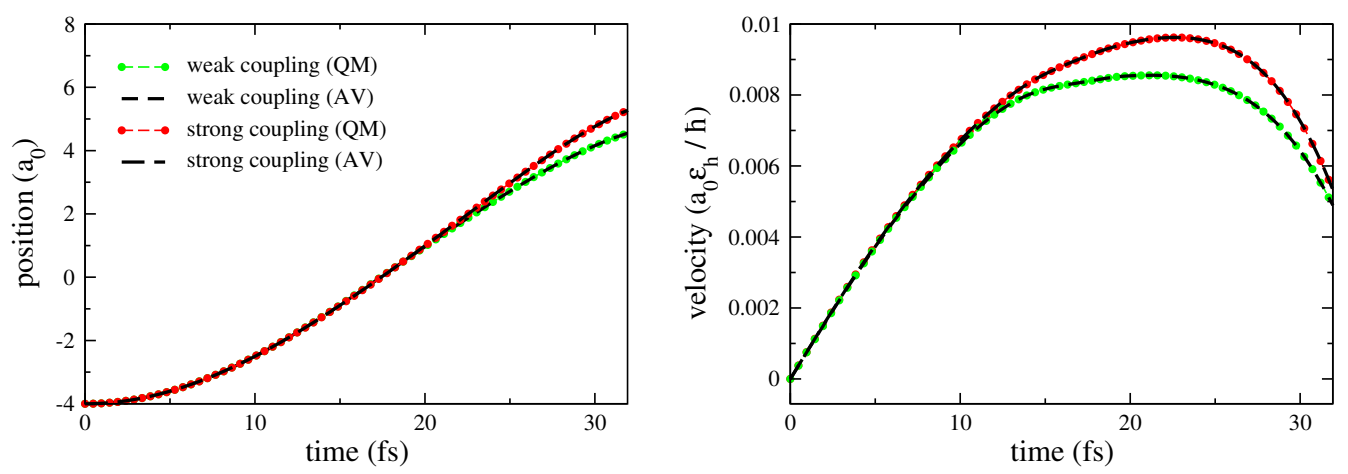

FIG. 10: Left: nuclear position as a function of time. Right: nuclear velocity as a function of time. The average position and velocity calculated from the quantum-mechanical (QM) propagation are shown as dotted red (strong coupling) and dotted green (weak coupling) lines. The long-dashed (strong coupling) and short-dashed (weak coupling) black lines are the results of classical propagation driven by the average force (AV) as in Eqs. (78) and (79).

position (left) and velocity (right) as functions of time from quantum-mechanical calculations, compared to the values of position and velocity of a classical particle moving according to the average force $\left\langle-\nabla_{R} \epsilon(R, t)\right\rangle_{\chi}$. As expected by the proof of the Ehrenfest theorem involving the nuclear wave-function $\chi(\underline{\underline{\mathbf{R}}}, t)$ and the nuclear Hamiltonian $\hat{H}_{n}$ presented in this section, the classical trajectory perfectly follows the evolution of the quantum mean values. In section IV, we 
studied the classical nuclear dynamics on the TDPES. However, we did not provide any argument on how that study can be associated with a classical limit of the nuclear motion that is able to, approximately, reproduce the expectation values of the nuclear position and momentum of the complete electron-nuclear system. Here, using the Ehrenfest theorem, we show how the nuclear position and momentum calculated from Eq. (44) can be linked to the expectation values of the nuclear position and momentum of the complete electron-nuclear system.

The Ehrenfest theorem [15] relates the time-derivative of the expectation value of a quantummechanical operator $\hat{O}$ to the expectation value of the commutator of that operator with the Hamiltonian, i.e.

$$
\frac{d}{d t}\langle\hat{O}(t)\rangle=\frac{1}{i \hbar}\langle[\hat{O}(t), \hat{H}]\rangle+\left\langle\partial_{t} \hat{O}(t)\right\rangle
$$

The second term on the RHS refers to the explicit time-dependence of $\hat{O}$. In particular, the theorem leads to the classical-like equations of motion for the mean value of position and momentum operators. For a system of electrons and nuclei, described by the Hamiltonian in Eq. (1) and the wave-function $\Psi(\underline{\underline{\mathbf{r}}}, \underline{\underline{\mathbf{R}}}, t)$, the mean values of the $\nu$-th nuclear position $\hat{\mathbf{R}}_{\nu}$ and momentum $\hat{\mathbf{P}}_{\nu}$ operators evolve according to the classical Hamilton's equations

$$
\begin{aligned}
\frac{d}{d t}\left\langle\hat{\mathbf{R}}_{\nu}\right\rangle_{\Psi} & =\frac{1}{i \hbar}\left\langle\left[\hat{\mathbf{R}}_{\nu}, \hat{H}(\underline{\underline{\mathbf{r}}}, \underline{\underline{\mathbf{R}}})\right]\right\rangle_{\Psi}=\frac{\left\langle\hat{\mathbf{P}}_{\nu}\right\rangle_{\Psi}}{M_{\nu}} \\
\frac{d}{d t}\left\langle\hat{\mathbf{P}}_{\nu}\right\rangle_{\Psi} & =\frac{1}{i \hbar}\left\langle\left[\hat{\mathbf{P}}_{\nu}, \hat{H}(\underline{\underline{\mathbf{r}}}, \underline{\underline{\mathbf{R}}})\right]\right\rangle_{\Psi}=\left\langle-\nabla_{\nu}\left(\hat{V}_{e n}(\underline{\underline{\mathbf{r}}}, \underline{\underline{\mathbf{R}}})+\hat{W}_{n n}(\underline{\underline{\mathbf{R}}})\right)\right\rangle_{\Psi} .
\end{aligned}
$$

Here, the operators do not depend explicitly on time and we indicate the integration over the full wave-function (electronic and nuclear coordinates) by $\langle\cdot\rangle_{\Psi}$. On the other hand, the nuclear equation (8) is a Schrödinger equation that contains a time-dependent vector potential and a timedependent scalar potential. Therefore, the Ehrenfest theorem for the nuclear subsystem reads

$$
\begin{aligned}
\frac{d}{d t}\left\langle\hat{\mathbf{R}}_{\nu}\right\rangle_{\chi} & =\frac{1}{i \hbar}\left\langle\left[\hat{\mathbf{R}}_{\nu}, \hat{H}_{n}(\underline{\underline{\mathbf{R}}})\right]\right\rangle_{\chi} \\
\frac{d}{d t}\left\langle\hat{\widetilde{\mathbf{P}}}_{\nu}\right\rangle_{\chi} & =\frac{1}{i \hbar}\left\langle\left[\hat{\widetilde{\mathbf{P}}}_{\nu}, \hat{H}_{n}(\underline{\underline{\mathbf{R}}})\right]\right\rangle_{\chi}+\left\langle\partial_{t} \mathbf{A}_{\nu}(\underline{\underline{\mathbf{R}}}, t)\right\rangle_{\chi}
\end{aligned}
$$

where [20]

$$
\hat{\tilde{\mathbf{P}}}_{\nu}=-i \hbar \nabla_{\nu}+\mathbf{A}_{\nu}(\underline{\underline{\mathbf{R}}}, t)
$$

is the expression of the nuclear canonical momentum operator in position representation, and

$$
\hat{H}_{n}(\underline{\underline{\mathbf{R}}})=\sum_{\nu=1}^{N_{n}} \frac{\left[-i \hbar \nabla_{\nu}+\mathbf{A}_{\nu}(\underline{\underline{\mathbf{R}}}, t)\right]^{2}}{2 M_{\nu}}+\epsilon(\underline{\underline{\mathbf{R}}}, t)
$$


is the nuclear Hamiltonian from Eq. (8). Note that the average operation is performed only on the nuclear wave-function as indicated by $\langle\cdot\rangle_{\chi}$. An explicit time-dependence appears in the expression of the momentum operator, due to the presence of the vector potential. This dependence is accounted for in the second term on the RHS of Eq. (67). While Eq. (66) is easily obtained from Eq. (64) by performing the integration over the electronic part of full wave-function, Eq. (67) is more involved and will be proved as follows. We rewrite LHS of Eq. 65) as

$$
\begin{aligned}
\frac{d}{d t}\left\langle\hat{\mathbf{P}}_{\nu}\right\rangle_{\Psi}= & \int d \underline{\underline{\mathbf{r}}} d \underline{\underline{\mathbf{R}}}\left[\Phi_{\underline{\underline{\mathbf{R}}}}^{*}(\underline{\underline{\mathbf{r}}}, t) \partial_{t} \chi^{*}(\underline{\underline{\mathbf{R}}}, t)+\chi^{*}(\underline{\underline{\mathbf{R}}}, t) \partial_{t} \Phi_{\underline{\underline{\mathbf{R}}}}^{*}(\underline{\underline{\mathbf{r}}}, t)\right] \hat{\mathbf{P}}_{\nu} \chi(\underline{\underline{\mathbf{R}}}, t) \Phi_{\underline{\mathbf{R}}}(\underline{\underline{\mathbf{r}}}, t) \\
& \left.+\int d \underline{\underline{\mathbf{r}}} d \underline{\underline{\mathbf{R}}} \chi^{*}(\underline{\underline{\mathbf{R}}}, t) \Phi_{\underline{\underline{\mathbf{R}}}}^{*} \underline{\underline{\mathbf{r}}}, t\right) \hat{\mathbf{P}}_{\nu}\left[\Phi_{\underline{\underline{\mathbf{R}}}}(\underline{\underline{\mathbf{r}}}, t) \partial_{t} \chi(\underline{\underline{\mathbf{R}}}, t)+\chi(\underline{\underline{\mathbf{R}}}, t) \partial_{t} \Phi_{\underline{\underline{\mathbf{R}}}}(\underline{\underline{\mathbf{r}}}, t)\right] .
\end{aligned}
$$

$\hat{\mathbf{P}}_{\nu}$ being a differential operator in position representation, its action on the factorized wavefunction is

$$
\hat{\mathbf{P}}_{\nu} \chi(\underline{\underline{\mathbf{R}}}, t) \Phi_{\underline{\underline{\mathbf{R}}}}(\underline{\underline{\mathbf{r}}}, t)=\left(\hat{\mathbf{P}}_{\nu} \chi(\underline{\underline{\mathbf{R}}}, t)\right) \Phi_{\underline{\underline{\mathbf{R}}}}(\underline{\underline{\mathbf{r}}}, t)+\chi(\underline{\underline{\mathbf{R}}}, t)\left(\hat{\mathbf{P}}_{\nu} \Phi_{\underline{\underline{\mathbf{R}}}}(\underline{\underline{\mathbf{r}}}, t)\right) .
$$

Then we use the nuclear equation (8) for

$$
\partial_{t} \chi(\underline{\underline{\mathbf{R}}}, t)=\frac{1}{i \hbar} \hat{H}_{n}(\underline{\underline{\mathbf{R}}}) \chi(\underline{\underline{\mathbf{R}}}, t)
$$

and its complex-conjugated $\left(\hat{H}_{n}(\underline{\underline{\mathbf{R}}})\right.$ is hermitian), the definition of the (real) vector potential

$$
\mathbf{A}_{\nu}(\underline{\underline{\mathbf{R}}}, t)=\int d \underline{\underline{\mathbf{r}}} \Phi_{\underline{\underline{\mathbf{R}}}}^{*}(\underline{\underline{\mathbf{r}}}, t) \hat{\mathbf{P}}_{\nu} \Phi_{\underline{\underline{\mathbf{R}}}}(\underline{\underline{\mathbf{r}}}, t)
$$

and the PNC, to derive

$$
\begin{aligned}
& \frac{d}{d t}\left\langle\hat{\mathbf{P}}_{\nu}\right\rangle_{\Psi}=\frac{1}{i \hbar} \int d \underline{\underline{\mathbf{R}}} \chi^{*}(\underline{\underline{\mathbf{R}}}, t)\left(\hat{\tilde{\mathbf{P}}}_{\nu} \hat{H}_{n}(\underline{\underline{\mathbf{R}}})-\hat{H}_{n}(\underline{\underline{\mathbf{R}}}) \hat{\tilde{\mathbf{P}}}_{\nu}\right) \chi(\underline{\underline{\mathbf{R}}}, t) \\
& \left.+\int d \underline{\underline{\mathbf{R}}}|\chi(\underline{\underline{\mathbf{R}}}, t)|^{2} \int d \underline{\underline{\mathbf{r}}}\left[\left(\partial_{t} \Phi_{\underline{\underline{\mathbf{R}}}}^{*}(\underline{\underline{\mathbf{r}}}, t)\right) \hat{\mathbf{P}}_{\nu} \Phi_{\underline{\underline{\mathbf{R}}}}(\underline{\underline{\mathbf{r}}}, t)+\Phi_{\underline{\underline{\mathbf{R}}}}^{*} \underline{\underline{\mathbf{r}}}, t\right) \hat{\mathbf{P}}_{\nu} \partial_{t} \Phi_{\underline{\mathbf{R}}}(\underline{\underline{\mathbf{r}}}, t)\right]
\end{aligned}
$$

with $\hat{\widetilde{\mathbf{P}}}_{\nu}=\hat{\mathbf{P}}_{\nu}+\mathbf{A}_{\nu}(\underline{\underline{\mathbf{R}}}, t)$. Using the relation

$$
\left.\left(\partial_{t} \Phi_{\underline{\underline{\mathbf{R}}}}^{*} \underline{\underline{\mathbf{r}}}, t\right)\right) \hat{\mathbf{P}}_{\nu} \Phi_{\underline{\underline{\mathbf{R}}}}(\underline{\underline{\mathbf{r}}}, t)=\partial_{t}\left(\Phi_{\underline{\underline{\mathbf{R}}}}^{*}(\underline{\underline{\mathbf{r}}}, t) \hat{\mathbf{P}}_{\nu} \Phi_{\underline{\underline{\mathbf{R}}}}(\underline{\underline{\mathbf{r}}}, t)\right)-\Phi_{\underline{\underline{\mathbf{R}}}}^{*}(\underline{\underline{\mathbf{r}}}, t) \hat{\mathbf{P}}_{\nu} \partial_{t} \Phi_{\underline{\underline{\mathbf{R}}}}(\underline{\underline{\mathbf{r}}}, t),
$$

for the term in the square brackets, leads to

$$
\frac{d}{d t}\left\langle\hat{\mathbf{P}}_{\nu}\right\rangle_{\Psi}=\int d \underline{\underline{\mathbf{R}}} \chi^{*}(\underline{\underline{\mathbf{R}}}, t)\left(\frac{1}{i \hbar}\left[\hat{\tilde{\mathbf{P}}}_{\nu}, \hat{H}_{n}(\underline{\underline{\mathbf{R}}})\right]+\partial_{t} \mathbf{A}_{\nu}(\underline{\underline{\mathbf{R}}}, t)\right) \chi(\underline{\underline{\mathbf{R}}}, t)
$$

recovering the term on the RHS of Eq. [67). A similar procedure [20] yields the relation

$$
\begin{aligned}
\left\langle\hat{\mathbf{P}}_{\nu}\right\rangle_{\Psi} & \left.=\int d \underline{\underline{\mathbf{r}}} d \underline{\underline{\mathbf{R}}} \Phi_{\underline{\underline{\mathbf{R}}}}^{*} \underline{\underline{\mathbf{r}}}, t\right) \chi^{*}(\underline{\underline{\mathbf{R}}}, t)\left[\left(\hat{\mathbf{P}}_{\nu} \chi(\underline{\underline{\mathbf{R}}}, t)\right) \Phi_{\underline{\underline{\mathbf{R}}}}(\underline{\underline{\mathbf{r}}}, t)+\chi(\underline{\underline{\mathbf{R}}}, t) \hat{\mathbf{P}}_{\nu} \Phi_{\underline{\mathbf{R}}}(\underline{\underline{\mathbf{r}}}, t)\right] \\
& =\int d \underline{\underline{\mathbf{R}}} \chi^{*}(\underline{\underline{\mathbf{R}}}, t)\left[\hat{\mathbf{P}}_{\nu}+\mathbf{A}_{\nu}(\underline{\underline{\mathbf{R}}}, t)\right] \chi(\underline{\underline{\mathbf{R}}}, t)=\left\langle\hat{\widetilde{\mathbf{P}}}_{\nu}\right\rangle_{\chi},
\end{aligned}
$$


which proves the identity of the LHSs of Eqs. (65) and (67).

We have proved the Ehrenfest theorem for the nuclear wave-function and nuclear Hamiltonian, deriving exact relations for the evolution of the mean values of nuclear position and momentum operators over the complete system. This outcome is consistent with the interpretation of $\chi(\underline{\underline{\mathbf{R}}}, t)$ as the proper nuclear wave-function that reproduces the nuclear density and current density of the complete system (see the discussion in section III).

In the one-dimensional system studied here, the gauge is chosen such that $A(R, t)=0$, therefore, the Ehrenfest equations become

$$
\begin{aligned}
\frac{d}{d t}\langle\hat{R}\rangle_{\chi} & =\frac{1}{i \hbar}\left\langle\left[\hat{R}, \hat{H}_{n}\right]\right\rangle_{\chi}=\frac{\langle\hat{P}\rangle_{\chi}}{M} \\
\frac{d}{d t}\langle\hat{P}\rangle_{\chi} & =\frac{1}{i \hbar}\left\langle\left[\hat{P}, \hat{H}_{n}\right]\right\rangle_{\chi}=\left\langle-\nabla_{R} \epsilon(R, t)\right\rangle_{\chi},
\end{aligned}
$$

where the mean force generating the classical-like evolution is determined as the expectation value, on the nuclear wave-function, of the gradient of the TDPES. If we replace the nuclear wavefunction in Eqs. (78) and (79) by a delta-function centered at the classical position, we get Eqs. (44) that was used in section IV to generate classical dynamics on the exact PES. That is why the classical nuclear dynamics on the TDPES could actually approximate the mean nuclear position and momentum.

We have numerically simulated classical dynamics under the following equations of motion

$$
\left\{\begin{array}{l}
\dot{R}=\frac{P}{M} \\
\dot{P}=\left\langle-\nabla_{R} \epsilon(R, t)\right\rangle_{\chi},
\end{array}\right.
$$

where $\epsilon(R, t)$ is obtained from the solution of the TDSE with Hamiltonian (14), for both sets of parameters producing strong and weak non-adiabatic coupling between the two lowest BO surfaces. The initial conditions for the classical evolution are exactly the initial mean position and mean velocity of the quantum particle. The results are shown in Fig. 11, where we plot the mean position (left) and velocity (right) as functions of time from quantum-mechanical calculations, compared to the values of position and velocity of a classical particle moving according to the average force $\left\langle-\nabla_{R} \epsilon(R, t)\right\rangle_{\chi}$. As expected by the proof of the Ehrenfest theorem involving the nuclear wavefunction $\chi(\underline{\mathbf{R}}, t)$ and the nuclear Hamiltonian $\hat{H}_{n}$ presented in this section, the classical trajectory perfectly follows the evolution of the quantum mean values. 

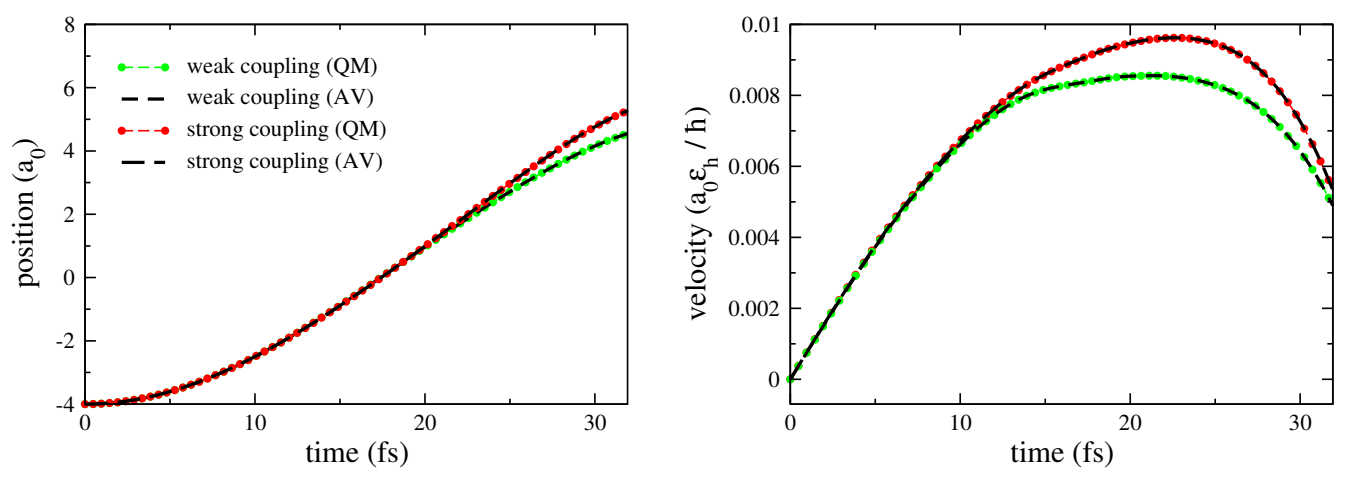

FIG. 11: Left: nuclear position as a function of time. Right: nuclear velocity as a function of time. The average position and velocity calculated from the quantum-mechanical (QM) propagation are shown as dotted red (strong coupling) and dotted green (weak coupling) lines. The long-dashed (strong coupling) and short-dashed (weak coupling) black lines are the results of classical propagation driven by the average force (AV) as in Eqs. (78) and (79).

\section{CONCLUSION}

In a system of interacting electrons and nuclei, the nuclear dynamics is fully determined by the TDPES and the time-dependent vector potential defined in the framework of the exact decomposition of the electronic and nuclear motions, as presented in this paper. We investigated some situations in which the vector potential can be gauged away, thus making the TDPES responsible for the nuclear evolution. This time-dependent scalar potential presents distinct and general features that can be analyzed in terms of its GI and GD components. The former, (i) in the region of an avoided crossing has a pronounced diabatic character, smoothly connecting different BOPESs along the direction of the nuclear wave-packet's motion, and, (ii) further away from the avoided crossing, dynamical steps appear between regions in which the (GI part of the) exact potential coincides with one or the other BOPES. The latter is either constant, if the nuclear wave-packet does not split, or stepwise constant, with the step at the same position, and with opposite slope, as in the GI part of the TDPES. We have analyzed in detail these features and discussed the connections with a classical picture of the nuclear evolution. To this end, we calculated the classical forces from the TDPES and from its GI component and performed classical nuclear dynamics driven by those forces. The importance of the GD part of the potential is evident as it improves the agreement of classical results with the quantum-mechanical calculations. We conclude that, if the exact TDPES is available, a single classical trajectory is able to reproduce quantum results fairly well, as long as quantum nuclear effects, such as tunneling or splitting of the nuclear wave- 
packet, are negligible. We have seen, in the example presented in the paper, that the splitting of the nuclear wave-function at the avoided crossing, that cannot be captured in the classical study, is responsible for the deviation of the classical results from the expected quantum behavior. Further analysis involving the propagation of multiple independent trajectories on the exact TDPES are envisaged. Such a multi-trajectory approach should be able to reproduce non-adiabatic effects, as those described above.

The development of mixed quantum-classical schemes to treat the non-adiabatic coupled electron-nuclear dynamics is still a challenging topic in physics and chemistry. Investigating the properties of the exact potential, that incorporates the effects of the electronic quantum dynamics on the nuclei, is a first step towards understanding the key features of approximated potentials and algorithms. We did not consider here cases where the vector potential cannot be gauged away. This will be the subject of future investigations.

In the final part of the paper, we have shown that the Ehrenfest theorem applied to calculate the mean nuclear position and momentum based on the nuclear equation alone reproduces the mean values calculated from the complete electron-nuclear system.

\section{Acknowledgements}

Partial support from the Deutsche Forschungsgemeinschaft (SFB 762) and from the European Commission (FP7-NMP-CRONOS) is gratefully acknowledged.

[1] M.Born and R.J. Oppenheimer, Annalen der Physik 389, 457 (1927).

[2] D. Polli, P. Altoè, O. Weingart, K.M. Spillane, C. Manzoni, D. Brida, G. Tomasello, G. Orlandi, P. Kukura, R.A. Mathies, M. Garavelli and G. Cerullo, Nature 467, 440 (2010).

[3] S. Hayashi, e. Tajkhorshid and K. Schulten, Biophys. J. 416, 403 (2009).

[4] W.C. Chung, S. Nanbu and T. Ishida, J. Phys. Chem. B 116, 8009 (2012).

[5] E. Tapavicza, A.M. Meyer and F. Furche, Phys. Chem. Chem. Phys. 13, 20986 (2011).

[6] T. Brixner, J. Stenger, H.M. Vaswani, M. Cho, R.E. Blankenship and G.R. Fleming, Nature 434, 625 (2005).

[7] C.A. Rozzi, S.M. Falke, N. Spallanzani, A. Rubio, E. Molinari, D. Brida, M. Maiuri, G. Cerullo, H. schramm, J. Christoffers and C. Lienau, Nat. Communic. 4, 1602 (2013).

[8] C. Silva, Nat. Mater. 12, 5 (2013). 
[9] A.E. Jailaubekov, A.P. Willard, J.R. Tritsch, W.L. Chan, N. Sai, R. Gearba, L.G. Kaake, K.J. Williams, K. Leung, P.J. Rossky and X.Y. Zhu, Nat. Mater. 12, 66 (2013).

[10] A.L. Sobolewski, W. Domcke, C. Dedonder-Lardeux and C. Jouvet, Phys. Chem. Chem. Phys. 4, 1093 (2002).

[11] M.T. do N. Varella, Y. Arasaki, H. Ushiyama, V. McKoy and K. Takatsukas, J. Chem. Phys. 124, 154302 (2006).

[12] J.Y. Fang and S. Hammes-Schiffer, J. Chem. Phys. 107, 8933 (1997).

[13] D. Marx, Chem. Phys. Chem. 7, 1848 (2006).

[14] J. Tully and R. Preston, J. Chem. Phys. 55, 562 (1971).

[15] P. Ehrenfest, Zeitschrift für Physik 45, 455 (1927).

[16] P. Pechukas, Phys. Rev. 181, 166 (1969).

[17] R. Kapral and G. Ciccotti, J. Chem.Phys. 110, 8916 (1999).

[18] A. Abedi, F. Agostini, Y. Suzuki and E.K.U. Gross, Phys. Rev. Lett 110, 263001 (2013).

[19] A. Abedi, N.T. Maitra and E.K.U. Gross, Phys. Rev. Lett. 105, 123002 (2010).

[20] A. Abedi, N.T. Maitra and E.K.U. Gross, J. Chem. Phys. 137, 22A530 (2012).

[21] J. Frenkel, Wave mechanics, Clarendon, Oxford ed. .

[22] S.K. Ghosh and A.K. Dhara, Phys. Rev. A 38, 1149 (1988).

[23] E. Runge and E.K.U. Gross, Phys. Rev. Lett. 52, 997 (1984).

[24] S. Shin and H. Metiu, J. Chem. Phys. 102, 23 (1995).

[25] M.D. Feit, F.A. Fleck Jr. and A. Steiger, J. Comput. Phys. 47, 412 (1982).

[26] The PNC is inserted in the calculation of the stationary variations of the quantum mechanical action by means of Lagrange multipliers.

[27] The scalar and vector potentials are uniquely determined up to within a gauge transformation, given in Eqs. (12) and (13). However, as expected, the nuclear Hamiltonian in Eq. (8) is form-invariant under such transformations.

[28] We reintroduce the bold-double underlined symbols for electronic and nuclear positions whenever the statements have general validity. 